\title{
Systematic Review \\ Efficacy and Safety of a Second Course of Stereotactic Radiation Therapy for Locally Recurrent Brain Metastases: A Systematic Review
}

\author{
François Lucia ${ }^{1}{ }^{*}$, Ruben Touati ${ }^{1}$, Nicolae Crainic ${ }^{2}$, Gurvan Dissaux ${ }^{1}$, Olivier Pradier ${ }^{1}$, Vincent Bourbonne ${ }^{1}$ D \\ and Ulrike Schick ${ }^{1}$ \\ 1 Radiation Oncology Department, University Hospital of Brest, 29200 Brest, France; \\ ruben.touati@chu-brest.fr (R.T.); gurvan.dissaux@chu-brest.fr (G.D.); olivier.pradier@chu-brest.fr (O.P.); \\ vincent.bourbonne@chu-brest.fr (V.B.); ulrike.schick@chu-brest.fr (U.S.) \\ 2 Neurology Department, University Hospital of Brest, 29200 Brest, France; nicolae.crainic@chu-brest.fr \\ * Correspondence: francois.lucia@chu-brest.fr
}

check for

updates

Citation: Lucia, F.; Touati, R.; Crainic, N.; Dissaux, G.; Pradier, O.;

Bourbonne, V.; Schick, U. Efficacy and

Safety of a Second Course of

Stereotactic Radiation Therapy for Locally Recurrent Brain Metastases: A Systematic Review. Cancers 2021, 13, 4929. https://doi.org/10.3390/ cancers13194929

Academic Editor: Johan Max Kros

Received: 31 August 2021

Accepted: 29 September 2021

Published: 30 September 2021

Publisher's Note: MDPI stays neutral with regard to jurisdictional claims in published maps and institutional affiliations.

Copyright: (c) 2021 by the authors. Licensee MDPI, Basel, Switzerland. This article is an open access article distributed under the terms and conditions of the Creative Commons Attribution (CC BY) license (https:/ / creativecommons.org/licenses/by/ $4.0 /)$.
Simple Summary: Approximately 30\% of patients diagnosed with cancer will ultimately develop brain metastases. Many improvements have been made in systemic and local cancer treatments, which have increased overall survival but also, as a consequence, the number of patients who present with local recurrence following intracranial stereotactic radiotherapy. The management of these recurrences remains controversial. The aim of our review is to evaluate the efficacy and tolerance of a second course of stereotactic radiotherapy.

Abstract: Recent advances in cancer treatments have increased overall survival and consequently, local failures (LFs) after stereotactic radiotherapy/radiosurgery (SRS/SRT) have become more frequent. LF following SRS or SRT may be treated with a second course of SRS (SRS2) or SRT (SRT2). However, there is no consensus on whenever to consider reirradiation. A literature search was conducted according to PRISMA guidelines. Analysis included 13 studies: 329 patients (388 metastases) with a SRS2 and 135 patients (161 metastases) with a SRT2. The 1-year local control rate ranged from $46.5 \%$ to $88.3 \%$. Factors leading to poorer LC were histology (melanoma) and lack of prior whole-brain radiation therapy, large tumor size and lower dose at SRS2/SRT2, poorer response at first SRS/SRT, poorer performance status, and no controlled extracranial disease. The rate of radionecrosis (RN) ranged from $2 \%$ to $36 \%$. Patients who had a large tumor volume, higher dose and higher value of prescription isodose line at SRS2/SRT2, and large overlap between brain volume irradiated at SRS1/SRT1 and SRS2/SRT2 at doses of 18 and 12 Gy had a higher risk of developing RN. Prospective studies involving a larger number of patients are still needed to determine the best management of patients with local recurrence of brain metastases

Keywords: control; local; metastasis; brain; radiosurgery; stereotactic radiotherapy; reirradiation; radionecrosis

\section{Introduction}

It is estimated that nearly $30 \%$ of cancer patients will develop brain metastases (BMs) during the clinical course of their illness [1], particularly patients with lung, melanoma, and breast cancer [2]. Alongside systemic treatments and surgery, the role of stereotactic radiosurgery (SRS) or fractionated stereotactic radiotherapy (SRT) for local control (LC) improvement is now well recognized. SRT is now preferred over whole-brain radiotherapy (WBRT) in order to maximize local control while minimizing the probability of neurocognitive decline [3,4], without compromising overall survival (OS). For patients with a limited number of BM, SRS/SRT achieves favorable tumor control rates of $80-90 \%$ at 12 months while sparing normal brain tissue, with a radionecrosis $(\mathrm{RN})$ rate of 3 to $5 \%[5,6]$. 
Recent advances in cancer systemic treatments, like targeted therapies and immune check point inhibitors, have increased OS and consequently, local failures (LFs) after SRS or SRT are observed more frequently [7-9]. The purpose of treatment for local recurrence of BM, particularly those that have undergone high-dose irradiation, is to improve the patient's quality of life by controlling local disease while minimizing the risk of significant toxicity [10]. The management of recurrent metastasis previously treated with SRS or SRT is, however, controversial [10]. Without treatment, the prognosis is poor, with survival often limited to weeks to months, usually due to neurological death [11]. Salvage options include systemic treatment, surgery, RT, or supportive care. Surgical resection may be considered as the preferred salvage modality to obtain histologic confirmation of recurrence and avoid reirradiation [12-16]. However, surgery is difficult if lesions are deeply located or in functional areas and has a significant rate of LF as stand-alone treatment [17]. WBRT alone does not achieve durable LC with a significant risk of neurocognitive sequelae [3]. In these patients, chemotherapy does not provide good results either, with median LC of $2 \pm 4$ months and OS of $3 \pm 7$ months [18-20]. In contrast, several studies have shown that repeated sessions of SRS/SRT can be performed with very good efficacy and tolerability for the management of new distant BM after a first course of SRS/SRT in order to defer WBRT [21,22]. In the last decade, because of these encouraging results and the lack of effective alternatives, a more frequent use of stereotactic reirradiation (SRS2/SRT2) has been noted in these patients given the major risk of adverse cognitive outcomes after WBRT. However, this approach is arguable both in terms of efficacy and tolerability. First, the effectiveness of a second radiotherapy treatment may be questioned if one considers local recurrence as arising from radio-resistant clones following previous radiotherapy [23]. In addition, SRS2/SRT2 could lead to a major risk of adverse events, most notably symptomatic RN given prior high-dose irradiation of the neighboring central nervous system (CNS) tissue [24]. Thus, it is essential to determine the optimal dosefractionation scheme for SRS2/SRT2. However, the lack of standardization on SRS and SRT protocols before the International Commission on Radiation Units and Measurements (ICRU) report 91 report made it difficult to compare results between studies performed at different centers [25].

We performed a systematic review of the literature to assess the efficacy and safety of a second course of SRS/SRT treatment in patients with local recurrence of one or more BMs after a first course of SRS/SRT.

\section{Materials and Methods}

\subsection{Selection Criteria and Search Strategy}

The study was performed according to the Preferred Reporting Items for Systematic Reviews and Meta-Analyzes (PRISMA) guidelines [26]. Because of the lack of a comparator in this setting, the Population, Intervention, Comparison, Outcome (PICO) model was not applicable. Suitable articles were identified by screening 3 electronic databases (Pubmed, Scopus, Cochrane Library) by 2 authors (F.L., R.T.) using the appropriate MeSH terms for the following search items: "brain," "encephalic," "cerebral" AND "stereotactic," "SRS," "SRT", "radiosurgery" AND "reirradiation," "re irradiation," "salvage," "repeated," "repeat" AND "metastases," "metastatic," "metastasis," "in-field relapse," "in-field progression," "local relapse," "local failure", "recurrent", and "in-field recurrence". Only studies evaluating the use of a second stereotactic radiotherapy of BM already irradiated with stereotactic radiotherapy were included in the final analysis. Only fully published, peer-reviewed articles in English, through February 1, 2021, were included in our analysis. Duplicate studies or those with insufficiently reported results were removed. To check compliance with the predetermined research criteria, an assessment was performed independently by 2 authors (V.B., N.C.). In the case of inconsistency or disagreement between the teams, a final decision was made with a third team (U.S., O.P.). The research methodology is shown in Figure 1. 


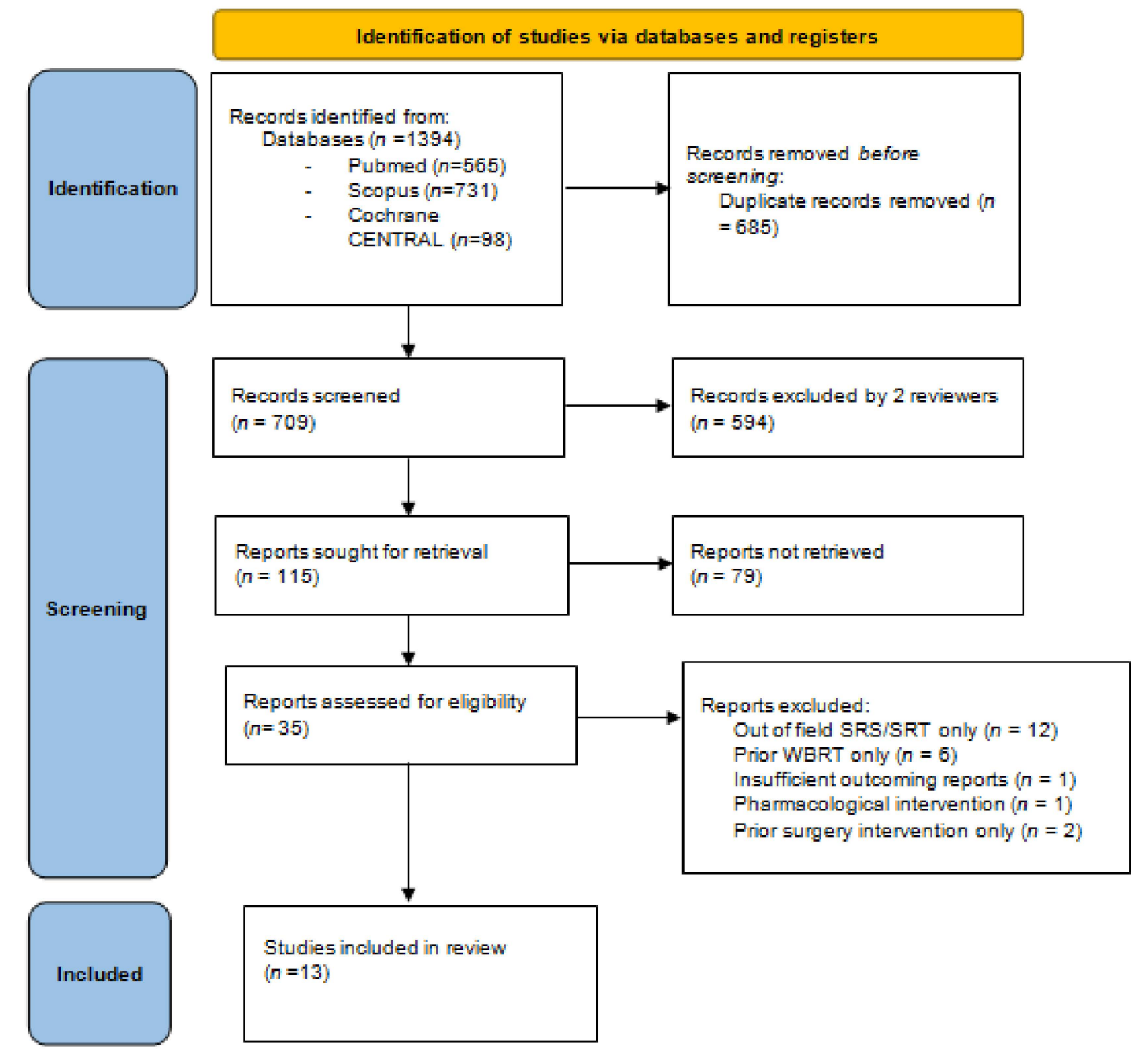

Figure 1. PRISMA 2020 flow diagram for our systematic review, which included searches of databases only.

\subsection{Validity Assessment}

For each individual study, risk of bias was assessed using the ROBINS-I tool [27] to estimate the following domains: (a) bias due to confounding of effects; (b) bias in selection of participants; (c) bias due to classification of interventions; (d) bias due to deviations from intended interventions; (e) bias due to missing data; (f) bias in measurement of the outcome; and $(\mathrm{g})$ bias in selection of the reported result. The categories for risk of bias judgements are "low risk", "moderate risk", "serious risk", and "critical risk" of bias. In some cases, no information on which to base a judgement about risk of bias for a domain was available.

\subsection{Data Extraction and Statistical Analysis}

Data extraction from the included articles was performed by one author (R.T.) and data accuracy was checked by a second author (F.L.). Patient, disease, and treatment characteristics, as well as LC rates and incidence of RN, were collected and reported using descriptive statistics. Study-specific 1-year OS values were pooled into a summary value and corresponding 95\% confidence intervals (CIs) by applying the "weighted median of medians" (MedCalc Software bvba, Ostend, Belgium; https://www.medcalc.org; accessed on 14 August 2015) method.

When not mentioned explicitly in the text, the 1-year OS was manually retrieved from Kaplan-Meier survival curves (available for all but 1 of the remaining studies). The study-specific proportion of patients with LF at 6 and 12 months of follow-up, or RN at 
any time after SRS2/SRT2, was pooled into summary proportions and corresponding 95\% CIs using random-effects models implemented in Medcalc. Heterogeneity between studies was quantified using the $\mathrm{I}^{2}$ statistic, which can be analyzed as the proportion of variability between estimates that is due to true heterogeneity rather than chance [28]. $\mathrm{I}^{2}$ values above $50 \%$ are considered to denote high heterogeneity. When this occurs, fitting meta-regression and subgroup analysis models (for continuous and categorical variables, respectively) is recommended to find study characteristics that correlate with the outcome and thus may explain some of the heterogeneity.

\section{Results}

The search workflow is outlined in Figure 1. Briefly, 1394 potential references were found. Thirty five full-text papers were initially included after checking for duplicates and pertinence. Among them, 22 were excluded due to a lack of prior SRS/SRT to the target metastasis (previous WBRT only or mixed interventions) or insufficient data reporting [21,28-48]. A total of 13 studies met the inclusion criteria and were analyzed in the final meta-analysis [49-61]. They included 464 patients and 549 treated metastases with a median follow-up of 11 (1-124) months by pooling patients from the 11 studies for which this data was available.

\subsection{Validity of Included Studies}

All included studies were retrospective. For each study, the risk of bias is reported in Table 1. Despite the presence of possible uncontrolled confounding factors, including differences in primary tumor type and systemic treatments, other local treatment already performed, different radiotherapy protocols at first and second SRS/SRT, and variability in age and performance status, we considered the risk of confounding bias low in all studies. Indeed, in all studies, the clinical and treatment variables were sufficiently precise to allow us to consider the level of risk as non-critical despite the presence of significant heterogeneity in the study population. It is difficult to exclude the possible presence of significant selection bias due to the retrospective nature of all studies. However, this risk is minimized by the use of precise criteria for the differential diagnosis between LF and RN (both at the time of the pre-radiotherapy assessment and in the post-treatment follow-up) in each cohort. All studies provided a precise description of the treatment procedures to avoid major classification bias. In addition, no major deviations from the planned treatment were reported in any study, especially with the short duration of the radiotherapy protocols. Overall, the risk of bias in measurement of the reported results was low thanks to the use of consensual diagnostic criteria, such as the Response Evaluation Criteria in Solid Tumors (RECIST) [53,54,56-58], Response Assessment in Neuro-Oncology (RANO)-BM [49,51], and Immunotherapy Response Assessment in Neuro-Oncology (iRANO) [52], or of a validated methodology, such as increased relative cerebral blood volume $>2$ (rCBV) extracted from perfusion magnetic resonance imaging (MRI) $[50,55,59]$. However, the variability in the imaging modality chosen and thus the diagnostic criteria used is of concern. Regarding the risk of bias for missing data, only four studies had short or unreported data, leading to a moderate level of risk. For the remaining articles reviewed, the level of risk was considered low because of the application of an adequate follow-up schedule with sufficient time to assess the efficacy and toxicity of radiation therapy $[51,53,56,60]$. Nevertheless, no study reported analytical strategies to account for loss of information. This lack of statistical strategies does not allow for concurrent events (death for extracranial progression) to be accounted for, despite the use of a clearly defined estimate of intervention outcome and toxicity, and makes the risk of selective reporting moderate for all studies. Finally, no study had a critical risk of bias, although it is important to note the inter-study variability, particularly with respect to the different modalities of outcome measurement. 
Table 1. Risk of bias.

\begin{tabular}{|c|c|c|c|c|c|c|c|}
\hline Study & $\begin{array}{l}\text { Confounding } \\
\text { of Effects }\end{array}$ & $\begin{array}{l}\text { Selection of } \\
\text { Participants }\end{array}$ & $\begin{array}{l}\text { Classification of } \\
\text { Interventions }\end{array}$ & $\begin{array}{c}\text { Deviations } \\
\text { from Intended } \\
\text { Interventions }\end{array}$ & $\begin{array}{c}\text { Missing } \\
\text { Data }\end{array}$ & $\begin{array}{c}\text { Measurement } \\
\text { of the } \\
\text { Outcome }\end{array}$ & $\begin{array}{c}\text { Selection of } \\
\text { the Reported } \\
\text { Result }\end{array}$ \\
\hline Terakedis 2013 [57] & + & + & + & + & + & + & ++ \\
\hline Greto 2014 [56] & + & + & + & + & ++ & + & ++ \\
\hline Holt 2015 [58] & + & + & + & + & + & ++ & ++ \\
\hline Minniti 2015 [55] & + & + & + & + & + & + & ++ \\
\hline Miyakawa 2016 [61] & + & + & + & + & + & ++ & ++ \\
\hline Kim 2017 [60] & + & + & + & + & ++ & + & ++ \\
\hline Koffer 2017 [59] & + & + & + & + & + & + & ++ \\
\hline McKay 2017 [54] & + & + & + & + & + & + & ++ \\
\hline Balermpas 2018 [52] & + & + & + & + & + & + & ++ \\
\hline Moreau 2018 [51] & + & + & + & + & ++ & + & ++ \\
\hline Dincoglan 2019 [50] & + & + & + & + & + & + & ++ \\
\hline Rana 2019 [53] & + & + & + & + & ++ & + & ++ \\
\hline Iorio Morin 2019 [62] & + & + & + & + & + & + & ++ \\
\hline
\end{tabular}

Risk of bias according the ROBINS-I tool; $(+)$ : low risk; $(++)$ : moderate risk.

\subsection{Clinical and Treatment-Related Characteristics}

Clinical and treatment-related characteristics for all studies are provided in Table 2, Table 3, Table 4, respectively. The median age, available for all studies, was 59 years (range 27-88). Ten studies reported Karnofsky Performance Status (KPS). The majority of patients were in good general condition with a median KPS of 85\% (60-100). All studies provided information on the type of primary tumor, but only 9 , including 279 patients, provided detailed data with a per patient basis. The most represented histologies were lung cancer $(108 / 279,38.8 \%)$, breast cancer $(61 / 279,21.9 \%)$, melanoma $(59 / 279,21.1 \%)$, and kidney cancer $(20 / 279,7.2 \%)$. The diagnosis of recurrence was mostly made by medical imaging. Only five studies reported pathological evaluation of metastases. It was performed only in 41 out of 121 metastases (33.9\%). In the remaining cases, diagnosis of recurrence was based on contrast-enhanced MRI (CE), perfusion MRI, perfusion MRI plus spectroscopy, and perfusion MRI plus DOTA-PET CT in five, two, three, and two studies, respectively. Ten studies reported possible surgical resection of some metastases at any time before SRS2/SRT2. It was performed in 89/458 (19.4\%) metastases, but residual disease was systematically present at the time of SRS2/SRT2. All studies reported the eventual possible use of WBRT: WBRT was administered at any time before SRS2/SRT2 in 98/464 (21\%). The median dose delivered at the time of the first course of SRS/SRT (SRS1/SRT1) was reported in 8/13 studies with a dose of 20 (18-24) Gy in 1 (1-3) fraction corresponding to a median biological effective dose (BED) of 60 Gy (50.4-81.6) assuming an $\alpha / \beta=10$. The median time between SRS1/SRT1 and SRS2/SRT2 was reported in all studies and was 13 (6-19) months. The median tumor volume at the time of reirradiation was 4.8 (1-40) cc. The median dose delivered at SRS2/SRT2 was 19 (15.5-26.5) Gy in 1 (1-3) fraction corresponding to a median BED of 50.4 (39-70.6) Gy assuming $\alpha / \beta=10$. A single-fraction regimen was used in the majority of cases: $70.7 \%(388 / 549)$. A multiple-fraction regimen was used in $29.3 \%(161 / 549)$ of metastases. 
Table 2. Summary of clinical characteristics.

\begin{tabular}{|c|c|c|c|c|c|c|c|c|c|c|c|c|c|c|c|c|c|c|}
\hline Study & $\begin{array}{l}\text { Number of } \\
\text { Patients }\end{array}$ & $\begin{array}{l}\text { Number } \\
\text { of } B M\end{array}$ & $\begin{array}{c}\text { Median } \\
\text { Age } \\
\text { (Range) }\end{array}$ & Male & Female & $\begin{array}{c}\text { Median } \\
\text { KPS } \\
\text { (Range) }\end{array}$ & $\begin{array}{l}\text { Lung } \\
\text { Tumor }\end{array}$ & $\begin{array}{l}\text { Breast } \\
\text { Tumor }\end{array}$ & $\mathrm{RCC}$ & Melanoma & Other & $\begin{array}{c}\text { Prior } \\
\text { Surgery }\end{array}$ & $\begin{array}{c}\text { Systemic } \\
\text { Treatment } \\
\text { Before }\end{array}$ & $\begin{array}{c}\text { Median } \\
\text { Number of } \\
\text { Metastases }\end{array}$ & $\begin{array}{c}\text { Metastases } \\
\text { Biopsy } \\
\text { Available }\end{array}$ & $\begin{array}{c}\text { Modality } \\
\text { Diagnostic }\end{array}$ & $\begin{array}{c}\text { Criteria } \\
\text { Diagnostic }\end{array}$ & $\begin{array}{c}\text { Median } \\
\text { Follow-Up }\end{array}$ \\
\hline $\begin{array}{l}\text { Terakedis } \\
2013 \text { [57] }\end{array}$ & 37 & 43 & $\begin{array}{c}51 \\
(27-84)\end{array}$ & 13 & 14 & NA & 9 & 8 & 2 & 20 & 4 & 13 & NA & 1 & NA & MRI & RECIST & $7(1-45)$ \\
\hline $\begin{array}{c}\text { Greto } \\
2014[56]\end{array}$ & 11 & 11 & $\begin{array}{c}47 \\
(33-77)\end{array}$ & NA & NA & $\begin{array}{c}80 \\
(60-100)\end{array}$ & 4 & 3 & NA & NA & 4 & NA & 11 & 1 & NA & MRI & RECIST & $4(1-7)$ \\
\hline $\begin{array}{c}\text { Holt 2015 } \\
{[58]}\end{array}$ & 13 & 15 & $\begin{array}{c}53 \\
(30-70)\end{array}$ & 5 & 8 & $\begin{array}{c}80 \\
(70-90)\end{array}$ & 1 & 2 & 1 & 9 & 2 & 15 & NA & 1 & 15 & NA & NA & $9(2.2-54.9)$ \\
\hline $\begin{array}{c}\text { Minniti } \\
2015 \text { [55] }\end{array}$ & 43 & 47 & 61 & 21 & 22 & $\begin{array}{c}80 \\
(60-100)\end{array}$ & 17 & 9 & NA & 11 & 6 & NA & NA & 1 & NA & $\begin{array}{c}\text { perfusion } \\
\text { MRI and } \\
\text { FDOPA } \\
\text { PET }\end{array}$ & $\mathrm{rCBV}>2$ & $19(2-27)$ \\
\hline $\begin{array}{l}\text { Miyakawa } \\
\text { 2016 [61] }\end{array}$ & 47 & 50 & $\begin{array}{c}61 \\
(40-85)\end{array}$ & 20 & 27 & $>70$ & 20 & 13 & 7 & NA & 7 & 0 & NA & 1 & 7 & $\begin{array}{c}\text { MRI + PET } \\
\text { methionine } \\
\text { ou biopsie } \\
\text { MRI spec- }\end{array}$ & NA & $10(1-40)$ \\
\hline$\underset{\text { [60] }}{\operatorname{Kim}} 2017$ & 84 & 108 & $\begin{array}{c}59.4 \\
\text { (mean) }\end{array}$ & 68 & 46 & NA & 79 & 10 & 15 & NA & 10 & 0 & NA & 1 & NA & $\begin{array}{l}\text { MRI spec- } \\
\text { troscopy, } \\
\text { perfusion } \\
\text { \& TEP }\end{array}$ & RECIST & NA \\
\hline $\begin{array}{c}\text { Koffer } \\
2017 \text { [59] }\end{array}$ & 22 & 24 & $\begin{array}{c}59 \\
(43-80)\end{array}$ & $\begin{array}{c}6 \\
(37 \%)\end{array}$ & $\begin{array}{c}16 \\
(64 \%)\end{array}$ & NA & 9 & 2 & 2 & 0 & 9 & 5 & NA & 1 & 5 & $\begin{array}{l}\text { perfusion, } \\
\text { spec- } \\
\text { troscopy, } \\
\text { PET }\end{array}$ & $\begin{array}{l}\mathrm{rCBV}>2 \\
\text { and cho/cr }\end{array}$ & 8.8 (NA) \\
\hline $\begin{array}{c}\text { McKay } \\
2017 \text { [54] }\end{array}$ & 32 & 46 & $\begin{array}{c}59 \\
(36-88)\end{array}$ & NA & NA & $\begin{array}{c}80 \\
(60-100)\end{array}$ & 16 & 9 & 2 & 2 & 3 & 23 & NA & 1 & 11 & $\begin{array}{l}\text { perfusion } \\
\text { MRI }\end{array}$ & RECIST & $24(12-124)$ \\
\hline $\begin{array}{c}\text { Moreau } \\
2018 \text { [51] }\end{array}$ & 30 & 36 & $\begin{array}{c}59 \\
(39-83)\end{array}$ & 20 & 10 & $\begin{array}{c}90-100 \\
(70-100)\end{array}$ & 15 & 5 & 0 & 4 & 6 & 3 & NA & 1 & 3 & $\begin{array}{l}\text { perfusion } \\
\text { MRI } \\
\text { MRI }\end{array}$ & RANO-BM & $14(1-107)$ \\
\hline $\begin{array}{l}\text { Dincoglan } \\
2019 \text { [50] }\end{array}$ & 30 & 30 & $\begin{array}{c}57 \\
(38-78)\end{array}$ & 16 & 14 & $\begin{array}{c}80 \\
(70-100)\end{array}$ & 11 & 9 & 3 & 4 & 3 & NA & $\mathrm{NA}$ & 1 & NA & $\begin{array}{c}\text { perfusion, } \\
\text { spec- } \\
\text { troscopy, } \\
\text { PET }\end{array}$ & $\begin{array}{c}\mathrm{rCBV}>2 \\
\mathrm{cho} / \mathrm{cr}\end{array}$ & $22(10-45)$ \\
\hline $\begin{array}{c}\text { Rana } 2019 \\
\text { [53] }\end{array}$ & 28 & 32 & 60 (NA) & 17 & 11 & $\begin{array}{c}80-100 \\
(70-100)\end{array}$ & 3 & 5 & 5 & 11 & 4 & 9 & 15 & 1 & NA & MRI & RECIST & NA \\
\hline $\begin{array}{c}\text { Iorio } \\
\text { Morin } \\
2019[62]\end{array}$ & 56 & 75 & $\begin{array}{c}57 \\
(27-81)\end{array}$ & 20 & 36 & 90 & 34 & 11 & 4 & 4 & 3 & 12 & 54 & 4 & NA & $\begin{array}{c}\text { MRI } \\
\text { perfusion, } \\
\text { spec- } \\
\text { troscopy, } \\
\text { PET }\end{array}$ & RANO-BM & 11 \\
\hline
\end{tabular}

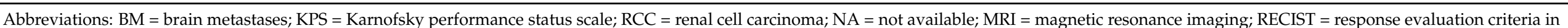

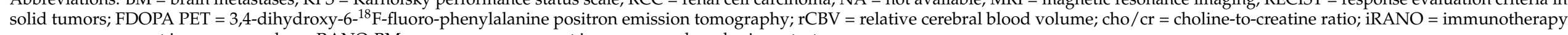
response assessment in neuro-oncology; RANO-BM = response assessment in neuro-oncology-brain metastases. 
Table 3. SRS1/SRT1 treatment characteristics.

\begin{tabular}{|c|c|c|c|c|c|c|c|c|c|}
\hline Study & WBRT & $\begin{array}{l}\text { Number } \\
\text { of SRS1 }\end{array}$ & $\begin{array}{l}\text { Number } \\
\text { of SRT1 }\end{array}$ & $\begin{array}{l}\text { Median } \\
\text { Volume cc }\end{array}$ & $\begin{array}{c}\text { Median Isodose } \\
\text { Line (\%) }\end{array}$ & $\begin{array}{c}\text { Median } \\
\text { Maximum } \\
\text { Dose SRS/SRT1 }\end{array}$ & $\begin{array}{c}\text { Median Maximum } \\
\text { Dose BED } \\
\text { SRS/SRT1 }\end{array}$ & $\begin{array}{l}\text { Median Margin } \\
\text { Dose SRS/SRT1 }\end{array}$ & $\begin{array}{c}\text { Median Margin } \\
\text { Dose BED } \\
\text { SRS/SRT1 }\end{array}$ \\
\hline Terakedis 2013 [57] & 17 & 43 & 0 & NA & 95 & 18.94 & 53.05 & 18 & 50.4 \\
\hline Holt 2015 [58] & 1 & 13 & 0 & $4.3(0.76-19.3)$ & 80 & $26.3(22.5-33.8)$ & $81.4(73.5-124.9)$ & $21(18-27)$ & $65.1(58.8-99.9)$ \\
\hline Minniti 2015 [55] & 0 & 47 & 0 & NA & NA & NA & NA & NA & NA \\
\hline Miyakawa 2016 [61] & 0 & 47 & 0 & $10.4(0.4-72.5)$ & 50 & 40 & 120 & 20 & $60 \mathrm{~Gy}$ \\
\hline Kim 2017 [60] & 0 & 108 & 0 & $3.89(0.024-25.5)$ & 50 & $37.8(24-48)$ & 109.24 & $18.9(12-24)$ & 54.6 \\
\hline McKay 2017 [54] & 8 & 46 & 0 & $1.28(0.01-22.6)$ & NA & NA & NA & $20(12-24)$ & $60(26.4-81.6)$ \\
\hline Balermpas 2018 [52] & 5 & 30 & 2 & $2.0(0.1-22.9)$ & $65(32-78)$ & $29.5(22.1-44.0)$ & $110(70.7-237.6)$ & $23.8(18.0-31.1)$ & $79.3(50.5-113.9)$ \\
\hline Moreau 2018 [51] & 24 & NA & NA & NA & NA & NA & NA & NA & NA \\
\hline Dincoglan 2019 [50] & 0 & 30 & 0 & $8.85(0.1-21.6)$ & $85-95$ & 20 & 56 & 18 (16-24 Gy) & 50.4 Gy \\
\hline Rana 2019 [53] & 8 & 30 & 2 & $0.48(0.02-6.70)$ & $83.5(69-96)$ & 28.74 & 97.72 & $24(18-30)$ & $81.6^{\circ}$ \\
\hline Iorio Morin 2019 [62] & 21 & 75 & 0 & $0.86(0.01-27.3)$ & $50(45-85)$ & $40(28-48)$ & $120(67.2-163.2)$ & $20(14-24)$ & $60(33.6-81.6)$ \\
\hline
\end{tabular}

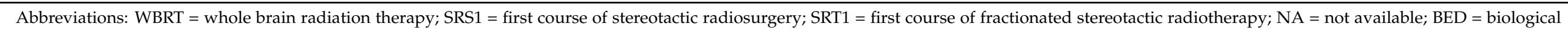
effective dose. 
Table 4. SRS2/SRT2 treatment characteristics.

\begin{tabular}{|c|c|c|c|c|c|c|c|c|c|}
\hline Study & $\begin{array}{c}\text { Median } \\
\text { Delay SRS1/SRT1 } \\
\text { to SRS2/SRT2 } \\
\text { (in Months) }\end{array}$ & $\begin{array}{l}\text { Number } \\
\text { of SRS2 }\end{array}$ & $\begin{array}{l}\text { Number } \\
\text { of SRT2 }\end{array}$ & $\begin{array}{l}\text { Median } \\
\text { Volume cc }\end{array}$ & $\begin{array}{l}\text { Median } \\
\text { Isodose } \\
\text { Line (\%) }\end{array}$ & $\begin{array}{c}\text { Median } \\
\text { Maximum } \\
\text { Dose SRS/SRT2 }\end{array}$ & $\begin{array}{c}\text { Median Maximum } \\
\text { Dose BED SRS/SRT2 }\end{array}$ & $\begin{array}{l}\text { Median Margin } \\
\text { Dose SRS/SRT2 }\end{array}$ & $\begin{array}{c}\text { Median Margin } \\
\text { Dose BED } \\
\text { SRS/SRT2 }\end{array}$ \\
\hline Greto 2014 [56] & $13(4-34)$ & 7 & 4 & $40.43(7-374)$ & $80(70-80)$ & 24.375 & 71.9 & $19.5(12-30)$ & 57.53 \\
\hline Holt 2015 [58] & $6.4(2.4-15.2)$ & 6 & 9 & $9.4(0.57-23)$ & 80 & $26.25(20-37.5)$ & 73.5 & $21(16-30)$ & 58.8 \\
\hline Minniti 2015 [55] & $17(6-56)$ & 0 & 47 & $12.3(1.5-33.1)$ & $85(80-90)$ & $50.8(42-50.8)$ & $35.7(35.7-43.2)$ & $24(21-24)$ & $43.2(35.7-43.2)$ \\
\hline Miyakawa 2016 [61] & $7.5(1-33)$ & 0 & 50 & $28.8(7.1-103)$ & 90 & 33.3 & 43.3 & 30 & 39 \\
\hline Koffer 2017 [59] & $13.4(1.9-52.4)$ & 24 & 0 & 3.3 & NA & NA & NA & $15.5(10-20)$ & $39.53(20-60)$ \\
\hline McKay 2017 [54] & $19(2-98)$ & 46 & 0 & $0.98(0.01-19.7)$ & NA & NA & NA & $20(14-22)$ & $60(33.6-70.4)$ \\
\hline Balermpas 2018 [52] & $12.4(3.2-88.2)$ & 24 & 8 & $2.5(0.1-37.5)$ & $69(53-80)$ & $28(17.4-38.1)$ & $97.2(40.1-126.3)$ & $23.5(14.3-33)$ & $70.6(34.5-89.9)$ \\
\hline Moreau 2018 [51] & $15.4(11-78)$ & 36 & 0 & $4.8(0.13-24.8)$ & 90 & 20 & 45.36 & $18(12-20)$ & 50.4 \\
\hline Dincoglan 2019 [50] & $13.5(3.7-49)$ & 0 & 30 & $14.6(1.6-35.6)$ & 85-95 & 23.33 & 39.7 & $21(21-30)$ & $35.7(35.7-48)$ \\
\hline Rana 2019 [53] & $9.7(2.5-56.9)$ & 19 & 13 & $1.35(0.11-34.9)$ & $83.5(69-96)$ & 31.73 & 77.96 & $26.5(18-36)$ & 65.1 \\
\hline Iorio Morin 2019 [62] & $13(3-47)$ & 75 & 0 & $1.19(0.07-20.6)$ & $50(30-80)$ & $100.8(52.8-120)$ & $36(24-40)$ & $18(12-20)$ & $50.4(26.4-60)$ \\
\hline
\end{tabular}

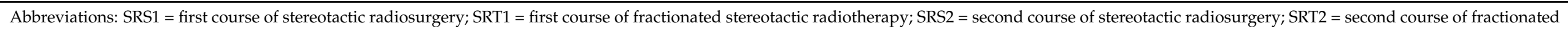
stereotactic radiotherapy; $\mathrm{NA}=$ not available; $\mathrm{BED}=$ biological effective dose. 


\subsection{Local Control and Overall Survival}

Data for 1-year OS were available in 11 studies. Pooling these results yielded a 1-year OS of $54.2 \%(95 \% \mathrm{CI}=38.9-69.1 \%)$. However, there was considerable heterogeneity $\mathrm{I}^{2}$ $=90.1 \%(95 \% \mathrm{CI}=84.3-93.8 \%)$ (Figure 2$)$. The only predictive factor found was a lower prescription dose in SRS2/SRT2 significantly associated with poorer survival in one study (Table 4).

Holt 2015

Minniti 2015

Miyakawa 2016

Kim 2017

Koffer 2017

McKay 2017

Balermpas 2018

Moreau 2018

Dincoglan 2019

Rana 2019

Iorio Morin 2019

Total (fixed effects)

Total (random effects)

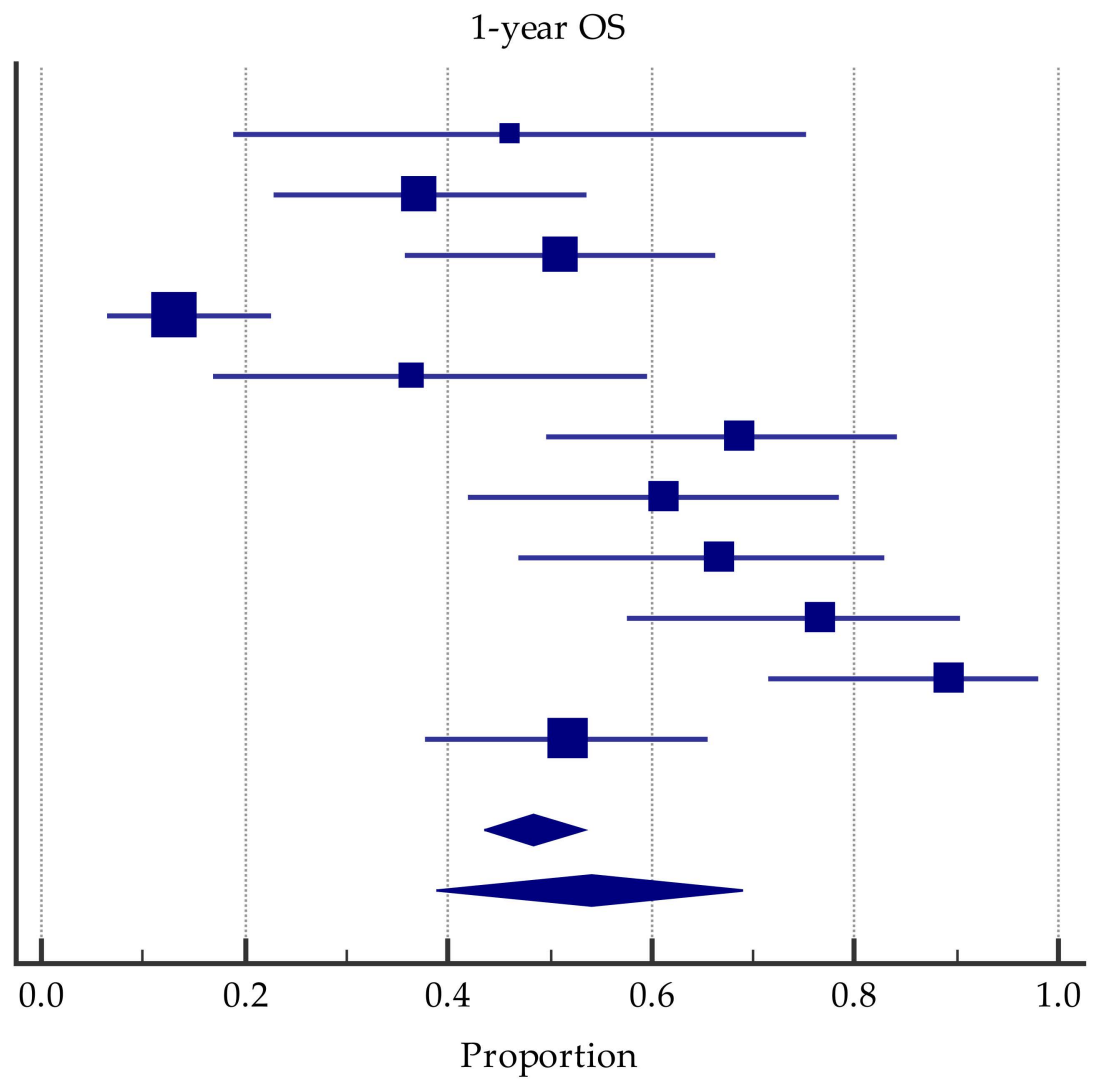

Figure 2. Forest plot of studies evaluating SRS2/SRT2: effect on 1-year overall survival.

In the pooled population of 11 studies, $\mathrm{LC}$ at 6 months was $88.4 \%(95 \% \mathrm{CI}=85.2-91.2 \%)$ with acceptable heterogeneity of $\mathrm{I}^{2}=0.0 \%(95 \% \mathrm{CI}=0.0-55.9 \%)$.

In the pooled population of 12 studies, $\mathrm{LC}$ at 1 year was $72.5 \%(95 \% \mathrm{CI}=64.6-79.7 \%)$ with significant heterogeneity of $\mathrm{I}^{2}=74.3 \%(95 \% \mathrm{CI}=54.6-85.5 \%)$ (Figure 3A). Eight studies reported 2-year LC, 54.2\% (95\%CI $=42.4-65.8 \%)$, with significant heterogeneity $\mathrm{I}^{2}=79.0 \%$ (C195 \% 58.9-89.2\%) (Figure 3B). Six studies reported predictive factors significantly associated with LC. Primary tumor type (melanoma versus other histologies), lack of prior WBRT, large tumor size at SRS2/SRT2, lower dose prescribed at SRS2/SRT2, poorer response at SRS1/SRT1, poorer KPS, and no controlled extracranial disease were factors associated with worse LC (Table 5). 
A

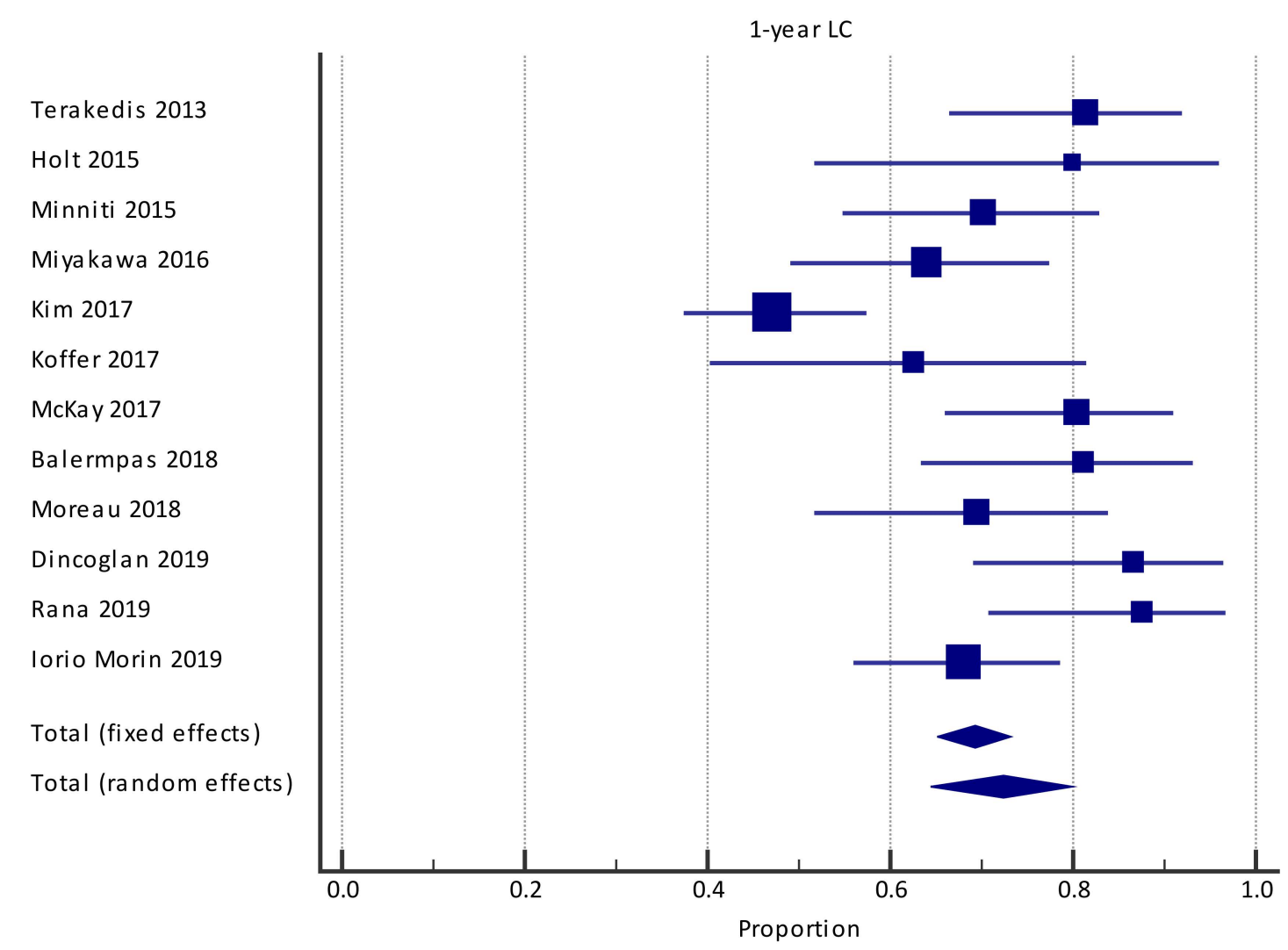

B

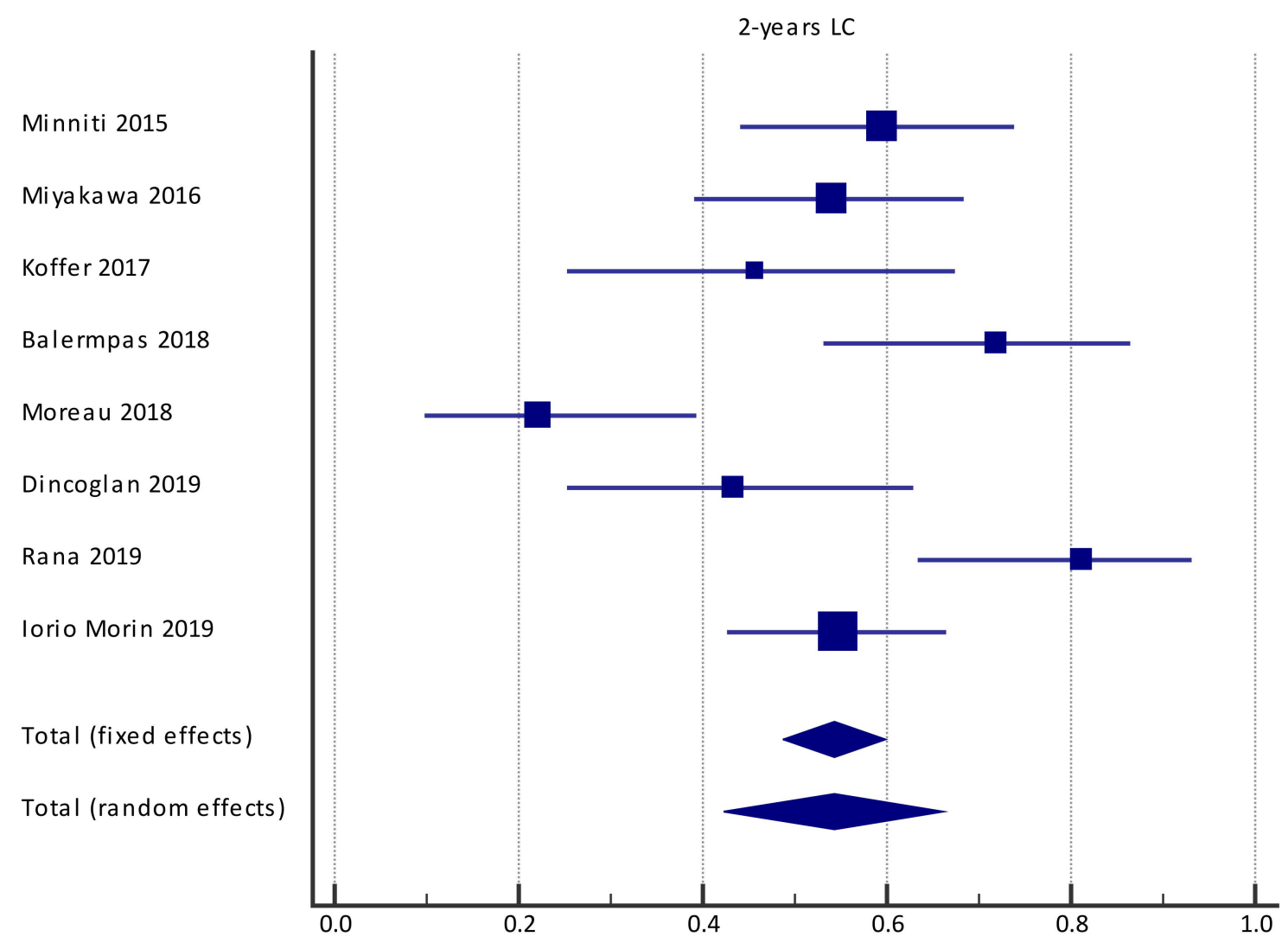

Figure 3. Forest plot of studies evaluating SRS2/SRT2: effect on 1-year local control (A) and 2-year local control (B). 
Table 5. Oncological endpoints and toxicities.

\begin{tabular}{|c|c|c|c|c|c|c|c|c|c|c|c|}
\hline Study & $\begin{array}{c}\text { 1-Year OS } \\
(\%)\end{array}$ & $\begin{array}{l}\text { 2-Years } \\
\text { OS (\%) }\end{array}$ & $\begin{array}{l}6 \text { Months } \\
\text { LC (\%) }\end{array}$ & $\underset{(\%)}{1-Y e a r ~ L C}$ & $\begin{array}{l}\text { 2-Years } \\
\text { LC (\%) }\end{array}$ & $\begin{array}{l}\text { Toxicity } \\
\text { Any } \\
\text { Grade (\%) }\end{array}$ & $\begin{array}{l}\text { Toxicity }> \\
2(\%)\end{array}$ & $\begin{array}{l}\text { RN Radio- } \\
\text { logical } \\
(\%)\end{array}$ & $\begin{array}{c}\text { Variables Related } \\
\text { to OS } \\
\text { [HR(95\%CI)] } \\
\end{array}$ & $\begin{array}{c}\text { Variables Related to LC } \\
\text { [HR(95\%CI)] }\end{array}$ & $\begin{array}{c}\text { Variables Related to RN } \\
\text { [HR(95\%CI)] }\end{array}$ \\
\hline $\begin{array}{l}\text { Terakedis } \\
2013[57]\end{array}$ & NA & NA & 83.3 & 80.6 & NA & NA & NA & 16 & NA & no & NA \\
\hline $\begin{array}{c}\text { Greto } 2014 \\
{[56]}\end{array}$ & NA & NA & NA & NA & NA & 15.4 & 0 & NA & NA & NA & NA \\
\hline $\begin{array}{l}\text { Holt 2015 } \\
{[58]}\end{array}$ & $\begin{array}{c}43.8 \\
(8.6-59.4)\end{array}$ & NA & 100 & $\begin{array}{c}75 \\
(31.5-93.1)\end{array}$ & NA & 15.4 & 15 & 15 & No & No & No \\
\hline Minniti & (0.0 & 20 & 91 & 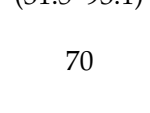 & 60 & 55 & 17 & 19 & NA & Melanoma [7.1 (1.9-21)] & $\begin{array}{c}\text {-Overlap } \\
\text { V18 } \\
\text { >RS1/SRT1 /V12 } 2 \text { SRS2/SRT2 } \\
\text { >10.1 }(1.1-13.6)]\end{array}$ \\
\hline $\begin{array}{l}\text { Miyakawa } \\
2016[61]\end{array}$ & 50 & 22 & 85 & 63 & 54 & 49 & NA & 17 & NA & NA & NA \\
\hline$\underset{[60]}{\operatorname{Kim} 2017}$ & 13.2 & NA & NA & 46.5 & NA & NA & NA & 1.8 & No & $\begin{array}{l}\text {-prescription radiation dose of } \\
16 \mathrm{~Gy}(p=0.000) ;- \text { tumor volume } \\
\text { less than both } 4 \mathrm{~mL}(p=0.001) \text { and } \\
10 \mathrm{~mL} \text { at SRS2/SRT2 }(p=0.008)\end{array}$ & NA \\
\hline $\begin{array}{c}\text { Koffer } \\
2017[59]\end{array}$ & 37.5 & 17.5 & 94.1 & 61.1 & 48 & NA & NA & 16 & No & $\begin{array}{c}\text { PTV size SRS2/SRT2 > 4cc } \\
\text { [NA(NA)] }\end{array}$ & $\begin{array}{c}\text { No (Trend for prior WBRT, } \\
\quad p=0.05 \text { ) }\end{array}$ \\
\hline $\begin{array}{l}\text { McKay } \\
2017[54]\end{array}$ & $70(55-88)$ & 0 & 90 & 79 (67-94) & NA & 35 & 11 & 36 & $\begin{array}{l}\text { Lower dose level } \\
\text { SRS2/SRT2 [0.64 } \\
\quad(0.49-0.84)]\end{array}$ & - & $\begin{array}{c}\text {-Tumor volume SRS2/SRT2 } \\
\text { [1.19 }(1.07-1.32)] \\
\text {-Dose SRS2/SRT2 [0.64 } \\
(0.48-0.84)] \\
\text { NB V40 } 4 \text { SRS/SRT1+2 }>0.76 \mathrm{CC} \\
=20 \% \text { NTCP }\end{array}$ \\
\hline $\begin{array}{c}\text { Balermpas } \\
2018[52]\end{array}$ & 61.7 & 46.3 & 92 & 79.5 & 71.5 & 19.4 & 12.9 & 16.1 & No & no & No \\
\hline $\begin{array}{l}\text { Moreau } \\
2018[51]\end{array}$ & $\begin{array}{c}65.5 \\
(47.3-80)\end{array}$ & $\begin{array}{c}27.6 \\
(14.7-45.7)\end{array}$ & $\begin{array}{c}82.9 \\
(67.6-91.9)\end{array}$ & $\begin{array}{c}67.8 \\
(51-81)\end{array}$ & 22 & 36 & 0 & 10 & No & $\begin{array}{c}\text { Prior WBRT [0.25 }(0.1-0.64)] \\
\text { PTV < 3cc [0.19 }(0.1-0.52)]\end{array}$ & no \\
\hline $\begin{array}{l}\text { Dincoglan } \\
2019[50]\end{array}$ & 76 & 34.9 & 93 & 86 & 44 & NA & 3.3 & 13 & NA & $\begin{array}{c}\text { PTV size SRS2/SRT2 > 20cc } \\
{[\text { NA(NA)] }}\end{array}$ & No \\
\hline $\begin{array}{c}\text { Rana } 2019 \\
{[53]}\end{array}$ & $\begin{array}{c}90.6 \\
(79-100)\end{array}$ & $\begin{array}{c}48.6 \\
(28.4-83.3)\end{array}$ & 90 & $\begin{array}{c}88.3 \\
(76.7-100)\end{array}$ & $\begin{array}{c}80.3 \\
(63.5-100)\end{array}$ & NA & NA & 18.8 & NA & no & $\begin{array}{c}\text {-Higher prescribed IDL } \\
\text { [HR0.886 (0.788-0.995)] } \\
\text {-Tumor Volume SRS2/SRT2 } \\
>0.48 \mathrm{cc}[1.55(1.05-2.29)]\end{array}$ \\
\hline
\end{tabular}


Table 5. Cont.

\begin{tabular}{|c|c|c|c|c|c|c|c|c|c|c|c|}
\hline Study & $\begin{array}{c}\text { 1-Year OS } \\
(\%)\end{array}$ & $\begin{array}{l}\text { 2-Years } \\
\text { OS (\%) }\end{array}$ & $\begin{array}{l}6 \text { Months } \\
\text { LC (\%) }\end{array}$ & $\begin{array}{c}\text { 1-Year LC } \\
(\%)\end{array}$ & $\begin{array}{l}\text { 2-Years } \\
\text { LC (\%) }\end{array}$ & $\begin{array}{c}\text { Toxicity } \\
\text { Any } \\
\text { Grade (\%) }\end{array}$ & $\begin{array}{l}\text { Toxicity }> \\
2(\%)\end{array}$ & $\begin{array}{c}\text { RN Radio- } \\
\text { logical } \\
(\%)\end{array}$ & $\begin{array}{c}\text { Variables Related } \\
\text { to OS } \\
\text { [HR( }(95 \% \mathrm{CI})]\end{array}$ & $\begin{array}{c}\text { Variables Related to LC } \\
\text { [HR(95\%CI)] }\end{array}$ & $\begin{array}{c}\text { Variables Related to RN } \\
\text { [HR(95\%CI)] }\end{array}$ \\
\hline $\begin{array}{l}\text { Iorio } \\
\text { Morin } \\
2019 \text { [62] }\end{array}$ & 52 & 37 & 85 & 68 & 55 & 5.0 & 1.3 & 4.0 & NA & $\begin{array}{c}\text { Higher Dose SRS2/SRT2 } \\
\text { [0.79(0.69-0.90)]; Best Response } \\
\text { SRS1/SRT1 } \\
\bullet \text { CR [0.026 (0.003-0.24)] } \\
\bullet \text { PR [0.062 (0.008-0.46)] } \\
\bullet \text { SD [0.090 (0.012-0.64)] } \\
\text {-KPS SRS2/SRT2 [0.93 (0.88-0.99)]; } \\
\text { Active primary tumor } \\
{[0.15(0.049-0.48)]}\end{array}$ & no \\
\hline
\end{tabular}

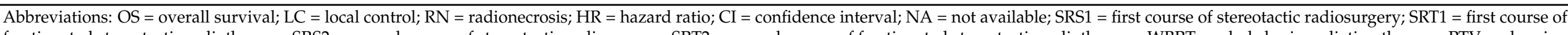

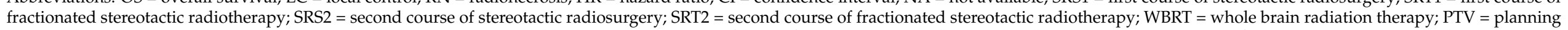
target volume; $\mathrm{CR}=$ complete response; $\mathrm{PR}=$ partial response; $\mathrm{SD}=$ stable disease; $\mathrm{KPS}=$ Karnofsky performance status scale; $\mathrm{IDL}=$ isodose line; $\mathrm{V}_{\mathrm{x}}=$ percentage of $\mathrm{CNS}$ receiving $\mathrm{x}$ Gy or higher. 
The majority of publications reviewed did not report sufficient information to be included in the meta-analysis.

\subsection{Radionecrosis}

Eleven studies reported the rate of $\mathrm{RN}$, with the cumulative rate yielding a crude median value of $14.3 \%(95 \% \mathrm{CI}=8.9-20.6 \%)$ in the pooled population at the end of the followup period, with significant heterogeneity $\left(\mathrm{I}^{2}=73.3 \%,(95 \% \mathrm{CI}=52.5-85.0 \%)\right.$ ) (Figure 4$)$. However, because no study reported either mean or total follow-up, it was not possible to assess the pooled estimate of incidence. Three studies reported significant risk factors for RN, large tumor volume at the time of SRS2/SRT2, high dose at the time of SRS2/SRT2, large overlap between brain volume irradiated at SRS1/SRT1 and SRS2/SRT2 at doses of 18 and 12 Gy, and a higher value of prescription isodose line at SRS2/SRT2 (Table 4). One study showed a trend toward increased risk with prior WBRT $(p=0.05)$.

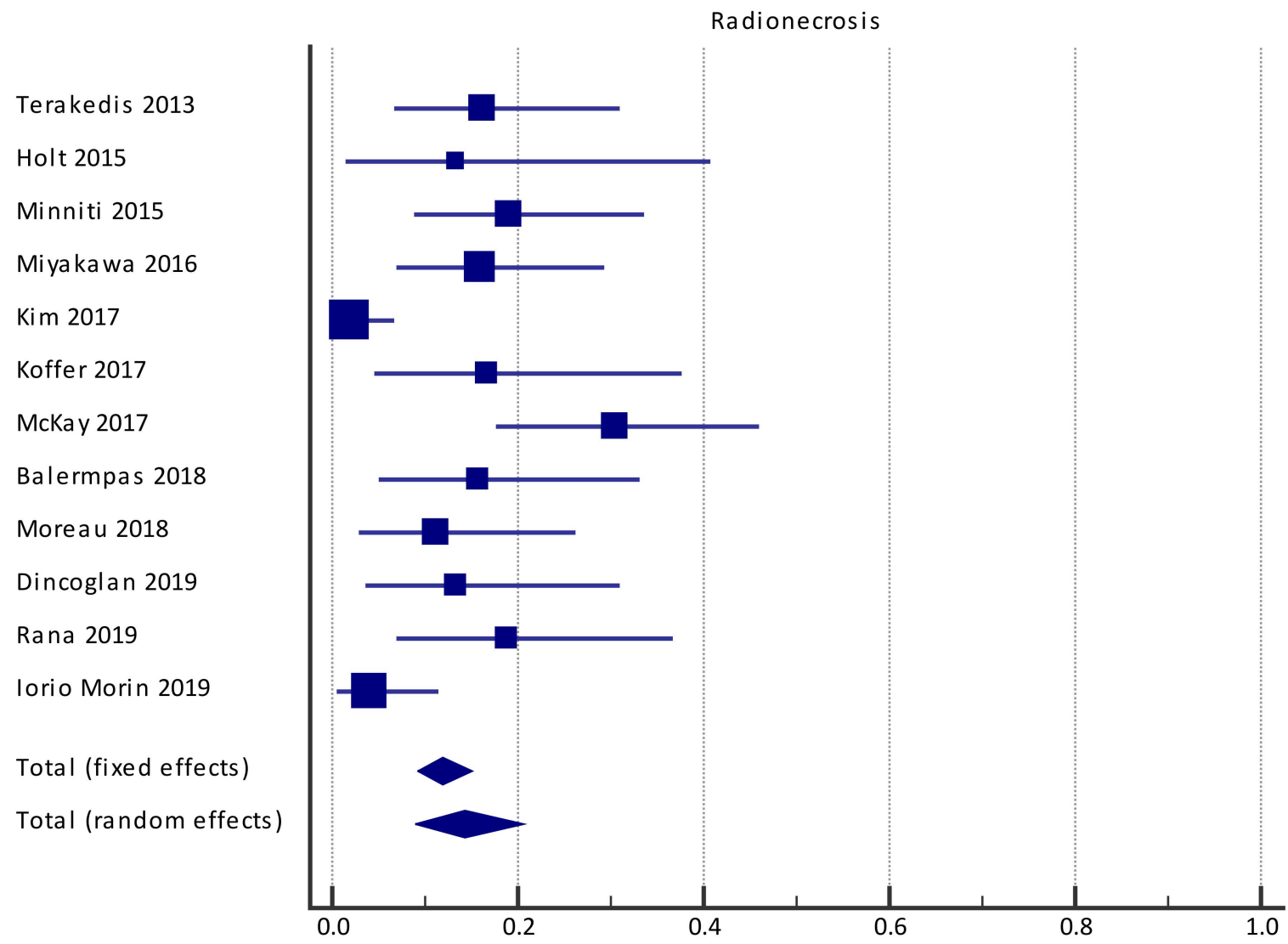

Figure 4. Forest plot of studies evaluating SRS2/SRT2: effect on radionecrosis.

The majority of publications reviewed did not report sufficient information to be included in the meta-analysis.

\section{Discussion}

There are limited data on the best salvage treatment strategy for in-site recurrent BM after initial SRS/SRT. Treatment options include surgical excision, systemic therapy, or re-irradiation with WBRT or SRS2/SRT2. The decision is often guided by a combination of factors including the patients' age and functional status, control of extracranial disease, intracranial tumor burden, prior treatments, type of primary cancer, and the possibility of targeted therapy [10]. Surgical approach is the preferred treatment option whenever possible to distinguish tumor recurrence from radionecrosis. This strategy provides widely varying results in terms of LC rates, ranging from $62 \%$ to $93 \%$ at one year $[12,13,15]$ and a 
median survival of 8.7 months [13]. However, reirradiation is often necessary to achieve better LC even with a neurosurgical approach [63]. Due to its invasiveness associated with a non-negligible risk of mortality and morbidity [12], the use of surgery is currently limited to selected cases, representing $1-11 \%$ of patients requiring salvage treatment for recurrent BM $[14,64]$. Reirradiation of CNS tumors has long been considered not advised because of the belief that normal brain tissue was at risk of irreversible tissue damage. In 1974, Shehata reported on the use of repeated WBRT in patients with progressive breast cancer [65]. However, WBRT increases the risk of subsequent cognitive impairment $[66,67]$ and compromises patient quality of life [3], particularly in long-term survivors who are oligometastatic or with only intracerebral progression. In addition, local recurrence after initial high-dose SRS/SRT treatment is often considered as a radioresistant lesion, so lower doses of WBRT than SRS/SRT are unlikely to achieve long-term disease control. Thus, radiation oncologists remain reluctant to reirradiate the CNS using conventional radiation therapy techniques. Localized irradiation, on the contrary, seems to be an interesting alternative since it may have a more acceptable toxicity profile. One approach to localized irradiation is SRS/SRT as an alternative. The reirradiation of a local target by SRS/SRT is of interest because it would provide better sparing of the healthy CNS compared to WBRT. This strategy has already shown its effectiveness and good tolerance in other pathologies, such as vestibular schwannomas [62] and meningiomas [68]. However, the prescribed doses are lower than those used for BM reirradiation.

In the present review, we investigated the use of a second course of SRS/SRT treatment for recurrent BM. We included 13 retrospective studies with a total of 464 patients and 549 treated metastases. Nine studies reported the histological type of the primary tumor, with a tendency to a higher proportion of melanoma and RCC than the known distribution in case of SRS1/SRT1. One explanation would be that brain metastases of melanoma and RCC are considered radioresistant $[69,70]$. Indeed, although the response rate to SRS/SRT is encouraging [71], LC seems inferior for these histological types and more particularly for melanoma ranging from 47 to $100 \%$ [71-73], while those for RCC range from 63 to $100 \%[71,72,74]$. The use of the ROBINS-I tool ensured that there was no critical bias in each domain for all included articles. LC is the primary objective of SRS/SRT, and our meta-analysis found a one-year LC rate of $72.5 \%$, which is close to the results reported by prospective trials on initial SRS/SRT $[5,75,76]$. Another interesting finding is that the 1-year OS in the pooled population was 54\% from the date of SRS2/SRT2. These data are similar to survival after a first course of SRS/SRT in selected subsets of patients with a high graded prognostic assessment (GPA) prognostic index score [74]. These figures are comparable to the results of surgical series in terms of LC $[12,13,15]$ and OS [13]. However, direct comparison between SRS2/SRT2 and resection is limited because the results regarding surgical excision are both for salvage treatment in case of local recurrence but also as a treatment for symptomatic or cortico-dependent RN [16]. Importantly, LC at 1 year and OS at 1 year in the pooled population showed significant heterogeneity between studies, with an $\mathrm{I}^{2}>50 \%$ for both endpoints. This result may be explained by a large disparity between studies on multiple prognostic factors (radiotherapy regimen, population included, systemic treatment, etc.). However, it may seem surprising that poorer KPS and lack of primary disease control can impact local control. Regarding KPS, one reason could be that these patients were no longer receiving systemic therapy. This information is not available in the relevant study [62]. Concerning the role of primary tumor control, one explanation could be that if the primary tumor is not controlled, it suggests that it is a more aggressive disease and resistant to any treatment and in particular to radiotherapy of brain metastases. Moreover, two studies showed a more important decrease of the LC at 2 years [50,51]. These results may be explained by the larger volumes of the treated metastases [50,51], which may have led to a lower median margin dose BED [50]. Unreported prognostic factors (number lost to follow-up, systemic treatment, and dosimetric parameters) could also explain this result. Another major finding of our review is the pooled rate of RN, estimated at $13 \%$ at a median follow-up of 11 months. Previous studies have found an 
increased risk of RN after SRS2/SRT2 of BM [24,55]. However, it should be noted that the incidence of $\mathrm{RN}$ is variable among the different salvage modalities of rescue therapy for recurrent BM after SRS/SRT. Rae et al. [64] investigated the risk of RN based on the salvage treatment type in patients with recurrent BM after SRS/SRT, excluding SRS2/SRT2. There was no significant increase in the incidence of $\mathrm{RN}$ in patients receiving salvage therapy, as the baseline rate of $\mathrm{RN}$ was $4.5 \%$ in patients with salvage therapy (regardless of salvage strategy) but increased to $21 \%$ in patients receiving simultaneous distant-site SRS/SRT and WBRT. Three studies (see Table 4 ) found three predictive factors significantly correlated with an increased risk of RN: (i) higher prescribed dose at the time of SRS2/SRT2 (two studies), (ii) larger tumor volume at the time of SRS2/SRT2 (two studies), and (iii) a larger area of overlap between the volumes of SRS1/SRT1 and SRS2/SRT2 (one study). A possible way to decrease the risk of toxicity with reirradiation is the use of fractionated stereotactic radiotherapy. In this case, the treatment is typically delivered in three to five sessions. Indeed, CNS tissues are known to be very sensitive to dose per fraction, so a small change in fractionation can potentially reduce the risk of $\mathrm{RN}[75,76]$. This approach is being evaluated in the initial treatment of large BM $[76,77]$. The role of fractionation in the course of reirradiation requires further study. In addition, there are no evidence-based OAR dose constraints. Indeed, multiple recommendations exist including American Association of Physicists in Medicine Task Group (AAPM-TG) 101 [78], Timmerman constraints [79] (updated in 2017 [80]), and the United Kingdom (UK) Consensus Guidelines [81] and HYTEC [82], but all of these recommendations have differences. This results in a large variability in the dose constraints used in the current trials, limiting the interpretation of toxicity rates or the acceptability of the dose constraints used [82].

The main limitations of our work are mainly its retrospective nature and the small number of eligible studies meeting our search criteria. In addition, there are different validated parameters for the differential diagnosis between progression and $\mathrm{RN}$ and the definition of response after SRS2/SRT2, but there is no standardized definition of these events. Thus, in the different series, different parameters were used, which represents a potential source of bias. This should be corrected with the consensus use of objective criteria, such as RANO-BM [83]. However, in cases of recurrent BM after salvage reirradiation, the diagnosis of LF and RN is extremely difficult [40]. Despite numerous diagnostic methods, including MR spectroscopy, perfusion $[84,85]$, and methionine positron emission tomography [86], the diagnosis remains difficult because of the overlapping radiographic appearance. Studies are ongoing to determine the best diagnostic strategy for differential diagnosis between radiation necrosis and BM relapse (NCT02636634; NCT04111588; NCT04244019). Finally, the lack of detailed reporting on multiple prognostic factors makes it difficult to determine their potential impact on observed outcomes. This mainly concerns systemic treatments and the great variability in the prescription of radiotherapy and its dose reports. Indeed, systemic treatments may have high intracranial efficacy, including next-generation tyrosine kinase inhibitors as well as immune checkpoint inhibitors, particularly in patients with asymptomatic disease for lung cancer, breast cancer, or melanoma [87]. Moreover, no studies consider the additional effect of systemic therapy. However, the authors considered that medical oncologists would not change the systemic treatment or start a new treatment after identifying a locally recurrent BM. In addition, they considered that pursuing the same systemic treatment had no effect on the outcome of SRS2/SRT2 $\mathrm{BM}$ because a local recurrence during this treatment can be a sign of an acquired drug resistance. The great variability of dose prescription (fractionation, prescription isodose, biological equivalent, etc.) in SRS/SRT was highlighted in the ICRU91 report [25]. It aimed to standardize the modalities of dose reporting in SRS/SRT, but it was published after the majority of the studies included in our review. Values of $\mathrm{I}^{2}$ above $50 \%$ for different outcomes are considered as denoting large heterogeneity. Thus, meta-regression and subgroup analysis models (for continuous and categorical variables, respectively) should have been performed to find study characteristics that might explain some of the heterogeneity. 
However, because of the lack of detail on the parameters that might be associated with the outcomes, these analyses could not be conducted.

Because of these different elements, no conclusions can be drawn on patient selection, neither the efficacy of SRS2/SRT2 nor the risk of RN.

Future studies should explore new strategies for recurrent BM, including different radiotherapy strategies (SRS or SRT), or treatments, such as the use of laser-induced thermotherapy [88] or the combination of several treatments (e.g., SRS/SRT and new systemic therapies), to reduce the risk of $\mathrm{RN}$ while improving local effectiveness. One study is currently investigating the combination of laser interstitial thermotherapy and pembrolizumab (NCT04187872).

\section{Conclusions}

A second SRS/SRT treatment (SRS2/SRT2) is an effective strategy for local recurrence of BM after initial SRS/SRT treatment, with similar results to surgical series in terms of overall survival and LC. However, SRS2/SRT2 results in symptomatic RN in 13\% of cases, a severe adverse event that can cause significant impairment of quality of life with physical or psychological impact. Unfortunately, as the available data are very heterogeneous and scarce, no reliable analysis looking for predictive factors for efficacy or toxicity could be performed. ICRU 91 standardization of dose ratios for stereotactic treatments will allow for better comparison of different protocols and their outcomes. Standardization of the criteria for differential diagnosis between local recurrence and RN seems necessary. Finally, real consideration should be given to the need for a second course of SRS in asymptomatic BM if high-efficacy intracranial systemic therapy is possible. Prospective studies involving a larger number of patients are still needed to determine the best management of patients with local recurrence of BM.

Author Contributions: Conceptualization, F.L.; methodology, F.L., R.T., N.C., G.D., O.P., V.B. and U.S.; software, R.T. and N.C.; validation, G.D., O.P., V.B. and U.S.; formal analysis, R.T.; investigation, F.L., R.T., N.C., G.D., O.P., V.B. and U.S.; data curation, F.L., R.T. and N.C.; writing-original draft preparation, F.L., and R.T.; writing - review and editing, N.C., G.D., O.P., V.B. and U.S.; visualization, G.D., O.P., V.B. and U.S. All authors have read and agreed to the published version of the manuscript.

Funding: This research received no external funding.

Institutional Review Board Statement: Not applicable.

Informed Consent Statement: Not applicable.

Data Availability Statement: Not applicable.

Conflicts of Interest: The authors declare no conflict of interest.

\section{References}

1. Mehta, M.; Tsao, M.N.; Whelan, T.J.; Morris, D.E.; Hayman, J.A.; Flickinger, J.; Mills, M.; Rogers, C.L.; Souhami, L. The American Society for Therapeutic Radiology and Oncology (ASTRO) evidence-based review of the role of radiosurgery for brain metastases. Int. J. Radiat. Oncol. 2005, 63, 37-46. [CrossRef]

2. Achrol, A.S.; Rennert, R.C.; Anders, C.; Soffietti, R.; Ahluwalia, M.S.; Nayak, L.; Peters, S.; Arvold, N.D.; Harsh, G.R.; Steeg, P.S.; et al. Brain metastases. Nat. Rev. Dis. Prim. 2019, 5, 5. [CrossRef]

3. Soffietti, R.; Kocher, M.; Abacioglu, U.; Villà, S.; Fauchon, F.; Baumert, B.G.; Fariselli, L.; Tzuk-Shina, T.; Kortmann, R.-D.; Carrie, C.; et al. A European Organisation for Research and Treatment of Cancer Phase III Trial of Adjuvant Whole-Brain Radiotherapy Versus Observation in Patients With One to Three Brain Metastases From Solid Tumors After Surgical Resection or Radiosurgery: Quality-of-Life Results. J. Clin. Oncol. 2013, 31, 65-72.

4. Brown, P.D.; Jaeckle, K.; Ballman, K.V.; Farace, E.; Cerhan, J.H.; Anderson, S.K.; Carrero, X.W.; Barker, F.G.; Deming, R.; Burri, S.H.; et al. Effect of Radiosurgery Alone vs Radiosurgery With Whole Brain Radiation Therapy on Cognitive Function in Patients with 1 to 3 Brain Metastases. JAMA 2016, 316, 401-409. [CrossRef]

5. Aoyama, H.; Shirato, H.; Tago, M.; Nakagawa, K.; Toyoda, T.; Hatano, K.; Kenjyo, M.; Oya, N.; Hirota, S.; Shioura, H.; et al. Stereotactic Radiosurgery Plus Whole-Brain Radiation Therapy vs Stereotactic Radiosurgery Alone for Treatment of Brain Metastases. JAMA 2006, 295, 2483-2491. [CrossRef] 
6. Sahgal, A.; Aoyama, H.; Kocher, M.; Neupane, B.; Collette, S.; Tago, M.; Shaw, P.; Beyene, J.; Chang, E.L. Phase 3 Trials of Stereotactic Radiosurgery With or Without Whole-Brain Radiation Therapy for 1 to 4 Brain Metastases: Individual Patient Data Meta-Analysis. Int. J. Radiat. Oncol. 2015, 91, 710-717. [CrossRef]

7. Larkin, J.; Sileni, V.C.; Gonzalez, R.; Grob, J.-J.; Cowey, C.L.; Lao, C.D.; Schadendorf, D.; Dummer, R.; Smylie, M.; Rutkowski, P.; et al. Combined Nivolumab and Ipilimumab or Monotherapy in Untreated Melanoma. N. Engl. J. Med. 2015, 373, 23-34. [CrossRef] [PubMed]

8. Reck, M.; Rodríguez-Abreu, D.; Robinson, A.G.; Hui, R.; Csőszi, T.; Fülöp, A.; Gottfried, M.; Peled, N.; Tafreshi, A.; Cuffe, S.; et al. Pembrolizumab versus Chemotherapy for PD-L1-Positive Non-Small-Cell Lung Cancer. N. Engl. J. Med. 2016, 375, 1823-1833. [CrossRef] [PubMed]

9. Duma, N.; Santana-Davila, R.; Molina, J.R. Non-Small Cell Lung Cancer: Epidemiology, Screening, Diagnosis, and Treatment. Mayo Clin. Proc. 2019, 94, 1623-1640. [CrossRef] [PubMed]

10. Ammirati, M.; Cobbs, C.S.; Linskey, M.E.; Paleologos, N.A.; Ryken, T.C.; Burri, S.H.; Asher, A.L.; Loeffler, J.S.; Robinson, P.D.; Andrews, D.W.; et al. The role of retreatment in the management of recurrent/progressive brain metastases: A systematic review and evidence-based clinical practice guideline. J. Neuro-Oncol. 2009, 96, 85-96. [CrossRef]

11. Wen, P.Y.; Loeffler, J.S. Management of brain metastases. Oncology 1999, 13, 941-954, 957-961; discussion 941-942, 949. [PubMed]

12. Kano, H.; Kondziolka, D.; Zorro, O.; Lobato-Polo, J.; Flickinger, J.; Lunsford, L.D. The results of resection after stereotactic radiosurgery for brain metastases. J. Neurosurg. 2009, 111, 825-831. [CrossRef]

13. Truong, M.T.; Clair, E.G.S.; Donahue, B.R.; Rush, S.C.; Miller, D.C.; Formenti, S.C.; Knopp, E.A.; Han, K.; Golfinos, J.G. Results of Surgical Resection for Progression of Brain Metastases Previously Treatedby Gamma Knife Radiosurgery. Neurosurgery 2006, 59, 86-97. [CrossRef]

14. Szeifert, G.T.; Atteberry, D.S.; Kondziolka, D.; Levivier, M.; Lunsford, L.D. Cerebral metastases pathology after radiosurgery. Cancer 2006, 106, 2672-2681. [CrossRef]

15. Vecil, G.G.; Suki, D.; Maldaun, M.V.C.; Lang, F.F.; Sawaya, R. Resection of brain metastases previously treated with stereotactic radiosurgery. J. Neurosurg. 2005, 102, 209-215. [CrossRef]

16. Jagannathan, J.; Bourne, T.D.; Schlesinger, D.; Yen, C.-P.; Shaffrey, M.E.; Laws, E.R.; Sheehan, J.P. Clinical and Pathological Characteristics of Brain Metastasis Resected After Failed Radiosurgery. Neurosurgery 2010, 66, 208-217. [CrossRef] [PubMed]

17. Kocher, M.; Soffietti, R.; Abacioglu, U.; Villà, S.; Fauchon, F.; Baumert, B.G.; Fariselli, L.; Tzuk-Shina, T.; Kortmann, R.-D.; Carrie, C.; et al. Adjuvant Whole-Brain Radiotherapy Versus Observation After Radiosurgery or Surgical Resection of One to Three Cerebral Metastases: Results of the EORTC 22952-26001 Study. J. Clin. Oncol. 2011, 29, 134-141. [CrossRef]

18. Christodoulou, C.; Bafaloukos, D.; Linardou, H.; Aravantinos, G.; Bamias, A.; Carina, M.; Klouvas, G.; Skarlos, D. Temozolomide (TMZ) combined with cisplatin (CDDP) in patients with brain metastases from solid tumors: A Hellenic Cooperative Oncology Group (HeCOG) Phase II study. J. Neuro-Oncol. 2005, 71, 61-65. [CrossRef]

19. Hwu, W.-J.; Lis, E.; Menell, J.H.; Panageas, K.S.; Lamb, L.A.; Merrell, J.; Williams, L.J.; Krown, S.E.; Livingston, P.O.; Wolchok, J.D.; et al. Temozolomide plus thalidomide in patients with brain metastases from melanoma. Cancer 2005, 103, 2590-2597. [CrossRef] [PubMed]

20. Kaba, S.; Kyritsis, A.P.; Hess, K.; Yung, W.K.; Mercier, R.; Dakhil, S.; Jaeckle, K.; Levin, V. TPDC-FuHu chemotherapy for the treatment of recurrent metastatic brain tumors. J. Clin. Oncol. 1997, 15, 1063-1070. [CrossRef] [PubMed]

21. Shultz, D.B.; Modlin, L.A.; Jayachandran, P.; Von Eyben, R.; Gibbs, I.C.; Choi, C.Y.; Chang, S.D.; Harsh, G.R.; Li, G.; Adler, J.R.; et al. Repeat Courses of Stereotactic Radiosurgery (SRS), Deferring Whole-Brain Irradiation, for New Brain Metastases After Initial SRS. Int. J. Radiat. Oncol. 2015, 92, 993-999. [CrossRef] [PubMed]

22. Yamanaka, K.; Iwai, Y.; Yasui, T.; Nakajima, H.; Komiyama, M.; Nishikawa, M.; Morikawa, T.; Kishi, H. Gamma Knife radiosurgery for metastatic brain tumor: The usefulness of repeated Gamma Knife radiosurgery for recurrent cases. Ster. Funct. Neurosurg. 1999, 72, 73-80. [CrossRef] [PubMed]

23. Kuwahara, Y.; Tomita, K.; Urushihara, Y.; Sato, T.; Kurimasa, A.; Fukumoto, M. Association between radiation-induced cell death and clinically relevant radioresistance. Histochem. Cell Biol. 2018, 150, 649-659. [CrossRef] [PubMed]

24. Sneed, P.K.; Mendez, J.; Hoek, J.G.M.V.-V.D.; Seymour, Z.A.; Ma, L.; Molinaro, A.M.; Fogh, S.E.; Nakamura, J.L.; McDermott, M.W. Adverse radiation effect after stereotactic radiosurgery for brain metastases: Incidence, time course, and risk factors. J. Neurosurg. 2015, 123, 373-386. [CrossRef] [PubMed]

25. Wilke, L.; Andratschke, N.; Blanck, O.; Brunner, T.; Combs, S.E.; Grosu, A.-L.; Moustakis, C.; Schmitt, D.; Baus, W.W.; Guckenberger, M. ICRU report 91 on prescribing, recording, and reporting of stereotactic treatments with small photon beams. Strahlenther. Onkol. 2019, 195, 193-198. [CrossRef]

26. Page, M.J.; McKenzie, J.; Bossuyt, P.M.; Boutron, I.; Hoffmann, T.C.; Mulrow, C.D.; Shamseer, L.; Tetzlaff, J.M.; Akl, E.; Brennan, S.; et al. The PRISMA 2020 statement: An updated guideline for reporting systematic reviews. BMJ 2021, 372, n71. [CrossRef]

27. Sterne, J.; Hernán, M.; Reeves, B.C.; Savović, J.; Berkman, N.D.; Viswanathan, M.; Henry, D.; Altman, D.G.; Ansari, M.T.; Boutron, I.; et al. ROBINS-I: A tool for assessing risk of bias in non-randomised studies of interventions. BMJ 2016, 355, i4919. [CrossRef]

28. Guan, Y.; Wang, C.; Zhu, H.; Li, J.; Xu, W.; Sun, L.; Pan, L.; Dai, J.; Wang, Y.; Wang, E.; et al. Hypofractionated Radiosurgery Plus Bevacizumab for Locally Recurrent Brain Metastasis with Previously High-Dose Irradiation. World Neurosurg. 2019, 133, e252-e258. [CrossRef] [PubMed] 
29. Davey, P.; O'Brien, P.F.; Schwartz, M.L.; Cooper, P.W. A phase I/II study of salvage radiosurgery in the treatment of recurrent brain metastases. Br. J. Neurosurg. 1994, 8, 717-723. [CrossRef] [PubMed]

30. Kwon, K.-Y.; Kong, D.-S.; Lee, J.-I.; Nam, D.-H.; Park, K.; Kim, J.H. Outcome of repeated radiosurgery for recurrent metastatic brain tumors. Clin. Neurol. Neurosurg. 2007, 109, 132-137. [CrossRef] [PubMed]

31. Noël, G.; Proudhom, M.-A.; Valery, C.-A.; Cornu, P.; Boisserie, G.; Hasboun, D.; Simon, J.M.; Feuvret, L.; Duffau, H.; Tep, B.; et al. Radiosurgery for re-irradiation of brain metastasis: Results in 54 patients. Radiother. Oncol. 2001, 60, 61-67. [CrossRef]

32. Bhatnagar, A.; Heron, D.E.; Kondziolka, D.; Lunsford, L.D.; Flickinger, J.C. Analysis of repeat stereotactic radiosurgery for progressive primary and metastatic CNS tumors. Int. J. Radiat. Oncol. 2002, 53, 527-532. [CrossRef]

33. Wowra, B.; Siebels, M.; Muacevic, A.; Kreth, F.W.; Mack, A.; Hofstetter, A. Repeated gamma knife surgery for multiple brain metastases from renal cell carcinoma. J. Neurosurg. 2002, 97, 785-793. [CrossRef] [PubMed]

34. Hillard, V.H.; Shih, L.L.; Chin, S.; Moorthy, C.R.; Benzil, D.L. Safety of multiple stereotactic radiosurgery treatments for multiple brain lesions. J. Neuro-Oncol. 2003, 63, 271-278. [CrossRef]

35. Shuto, T.; Fujino, H.; Inomori, S.; Nagano, H. Repeated gamma knife radiosurgery for multiple metastatic brain tumours. Acta Neurochir. 2004, 146, 989-993. [CrossRef]

36. Maranzano, E.; Trippa, F.; Casale, M.; Costantini, S.; Anselmo, P.; Carletti, S.; Principi, M.; Caserta, C.; Loreti, F.; Giorgi, C. Reirradiation of brain metastases with radiosurgery. Radiother. Oncol. 2012, 102, 192-197. [CrossRef]

37. Kim, D.H.; Schultheiss, T.E.; Radany, E.H.; Badie, B.; Pezner, R.D. Clinical outcomes of patients treated with a second course of stereotactic radiosurgery for locally or regionally recurrent brain metastases after prior stereotactic radiosurgery. J. Neuro-Oncol. 2013, 115, 37-43. [CrossRef] [PubMed]

38. Kurtz, G.; Zadeh, G.; Gingras-Hill, G.; Millar, B.-A.; Laperriere, N.; Bernstein, M.; Jiang, H.; Ménard, C.; Chung, C. Salvage Radiosurgery for Brain Metastases: Prognostic Factors to Consider in Patient Selection. Int. J. Radiat. Oncol. 2014, 88, 137-142. [CrossRef] [PubMed]

39. Iii, E.A.M.; Bhatnagar, J.P.; Xu, Y.; Arai, Y.; Niranjan, A.; Huq, M.S.; Lunsford, L.D. Evaluation of Tumor Progression and Detection of New Tumors during Repeat Gamma Knife ${ }^{\circledR S}$ Stereotactic Radiosurgery Utilizing the Co-Registration Tool in Leksell Gamma Plan®: Technical Note. Ster. Funct. Neurosurg. 2014, 92, 300-305.

40. Yomo, S.; Hayashi, M. Salvage stereotactic radiosurgery with adjuvant use of bevacizumab for heavily treated recurrent brain metastases: A preliminary report. J. Neuro-Oncol. 2015, 127, 119-126. [CrossRef]

41. Bates, J.; Youn, P.; Usuki, K.Y.; Dhakal, S.; Milano, M.T. Repeat courses of SRS in patients initially treated with SRS alone for brain-metastatic melanoma. Melanoma Manag. 2016, 3, 97-104. [CrossRef] [PubMed]

42. Shen, C.J.; Rigamonti, D.; Redmond, K.J.; Kummerlowe, M.N.; Lim, M.K.; Kleinberg, L.R. The strategy of repeat stereotactic radiosurgery without whole brain radiation treatment for new brain metastases: Outcomes and implications for follow-up monitoring. Pract. Radiat. Oncol. 2016, 6, 409-416. [CrossRef] [PubMed]

43. Huang, Z.; Sun, B.; Shen, G.; Cha, L.; Meng, X.; Wang, J.; Zhou, Z.; Wu, S. Brain metastasis reirradiation in patients with advanced breast cancer. J. Radiat. Res. 2017, 58, 142-148. [CrossRef] [PubMed]

44. Koiso, T.; Yamamoto, M.; Kawabe, T.; Watanabe, S.; Sato, Y.; Higuchi, Y.; Yamamoto, T.; Matsumura, A.; Kasuya, H. Follow-up results of brain metastasis patients undergoing repeat Gamma Knife radiosurgery. J. Neurosurg. 2016, 125, 2-10. [CrossRef]

45. Maranzano, E.; Terenzi, S.; Anselmo, P.; Casale, M.; Arcidiacono, F.; Loreti, F.; Di Marzo, A.; Draghini, L.; Italiani, M.; Trippa, F. A prospective phase II trial on reirradiation of brain metastases with radiosurgery. Clin. Transl. Radiat. Oncol. 2019, 17, 1-6. [CrossRef]

46. Suzuki, R.; Wei, X.; Allen, P.K.; Welsh, J.W.; Cox, J.D.; Komaki, R.; Lin, S.H. Outcomes of re-irradiation for brain recurrence after prophylactic or therapeutic whole-brain irradiation for small cell lung Cancer: A retrospective analysis. Radiat. Oncol. 2018, 13, 258. [CrossRef]

47. Jablonska, P.; Tejero, D.S.; González, A.C.; Morales, M.G.; Moreno, L.A.; Moreno-Jiménez, M.; García-Consuegra, A.; Pastor, S.M.; Echavarri, P.D.; Gil-Bazo, I.; et al. Repeated stereotactic radiosurgery for recurrent brain metastases: An effective strategy to control intracranial oligometastatic disease. Crit. Rev. Oncol. 2020, 153, 103028. [CrossRef]

48. Telentschak, S.; Ruess, D.; Grau, S.; Goldbrunner, R.; von Spreckelsen, N.; Jablonska, K.; Treuer, H.; Kocher, M.; Ruge, M. Cyberknife ${ }^{\circledR}$ hypofractionated stereotactic radiosurgery (CK-hSRS) as salvage treatment for brain metastases. J. Can. Res. Clin. Oncol. 2021, 147, 2765-2773. [CrossRef]

49. Iorio-Morin, C.; Mercure-Cyr, R.; Figueiredo, G.; Touchette, C.J.; Masson-Côté, L.; Mathieu, D. Repeat stereotactic radiosurgery for the management of locally recurrent brain metastases. J. Neuro-Oncol. 2019, 145, 551-559. [CrossRef]

50. Dincoglan, F.; Sager, O.; Demiral, S.; Gamsiz, H.; Uysal, B.; Onal, E.; Ekmen, A.; Dirican, B.; Beyzadeoglu, M. Fractionated stereotactic radiosurgery for locally recurrent brain metastases after failed stereotactic radiosurgery. Indian J. Cancer 2019, 56, 151-156. [CrossRef]

51. Moreau, J.; Khalil, T.; Dupic, G.; Chautard, E.; Lemaire, J.-J.; Magnier, F.; Dedieu, V.; Lapeyre, M.; Verrelle, P.; Biau, J. Second course of stereotactic radiosurgery for locally recurrent brain metastases: Safety and efficacy. PLoS ONE 2018, 13, e0195608. [CrossRef] [PubMed]

52. Balermpas, P.; Stera, S.; Von Der Grün, J.M.; Loutfi-Krauss, B.; Forster, M.-T.; Wagner, M.; Keller, C.; Rödel, C.; Seifert, V.; Blanck, O.; et al. Repeated in-field radiosurgery for locally recurrent brain metastases: Feasibility, results and survival in a heavily treated patient cohort. PLoS ONE 2018, 13, e0198692. 
53. Rana, N.; Pendyala, P.; Cleary, R.K.; Luo, G.; Zhao, Z.; Chambless, L.B.; Cmelak, A.J.; Attia, A.; Stavas, M.J. Long-term Outcomes after Salvage Stereotactic Radiosurgery (SRS) following In-Field Failure of Initial SRS for Brain Metastases. Front. Oncol. 2017, 7, 279. [CrossRef]

54. McKay, W.H.; Mctyre, E.R.; Okoukoni, C.; Alphonse-Sullivan, N.K.; Ruiz, J.; Munley, M.T.; Qasem, S.; Lo, H.-W.; Xing, F.; Laxton, A.W.; et al. Repeat stereotactic radiosurgery as salvage therapy for locally recurrent brain metastases previously treated with radiosurgery. J. Neurosurg. 2017, 127, 148-156. [CrossRef]

55. Minniti, G.; Scaringi, C.; Paolini, S.; Clarke, E.; Cicone, F.; Esposito, V.; Romano, A.; Osti, M.; Enrici, R.M. Repeated stereotactic radiosurgery for patients with progressive brain metastases. J. Neuro-Oncol. 2015, 126, 91-97. [CrossRef]

56. Greto, D.; Livi, L.; Bonomo, P.; Masi, L.; Detti, B.; Meattini, I.; Mangoni, M.; Doro, R.; Favuzza, V.; Cipressi, S.; et al. Cyberknife stereotactic radiosurgery for the re-irradiation of brain lesions: A single-centre experience. Radiol. Med. 2014, 119, 721-726. [CrossRef] [PubMed]

57. Terakedis, B.E.; Jensen, R.L.; Boucher, K.; Shrieve, D.C. Tumor control and incidence of radiation necrosis after reirradiation with stereotactic radiosurgery for brain metastases. J. Radiosurg. SBRT 2013, 3, 21-28.

58. Holt, D.E.; Gill, B.S.; Clump, D.A.; Leeman, J.E.; Burton, S.A.; Amankulor, N.M.; Engh, J.A.; Heron, D.E. Tumor Bed Radiosurgery Following Resection and Prior Stereotactic Radiosurgery for Locally Persistent Brain Metastasis. Front. Oncol. 2015, 5, 84. [CrossRef] [PubMed]

59. Koffer, P.; Chan, J.; Rava, P.; Gorovets, D.; Ebner, D.; Savir, G.; Kinsella, T.; Cielo, D.; Hepel, J.T. Repeat Stereotactic Radiosurgery for Locally Recurrent Brain Metastases. World Neurosurg. 2017, 104, 589-593. [CrossRef]

60. Kim, I.-Y.; Jung, S.; Jung, T.-Y.; Moon, K.-S.; Jang, W.-Y.; Park, J.-Y.; Song, T.-W.; Lim, S.-H. Repeat Stereotactic Radiosurgery for Recurred Metastatic Brain Tumors. J. Korean Neurosurg. Soc. 2018, 61, 633-639. [CrossRef]

61. Miyakawa, A.; Shibamoto, Y.; Takemoto, S.; Serizawa, T.; Otsuka, S.; Hirai, T. Fractionated stereotactic radiotherapy for metastatic brain tumors that recurred after gamma knife radiosurgery results in acceptable toxicity and favorable local control. Int. J. Clin. Oncol. 2016, 22, 250-256. [CrossRef] [PubMed]

62. Iorio-Morin, C.; Liscak, R.; Vladyka, V.; Kano, H.; Jacobs, R.C.; Lunsford, L.D.; Cohen-Inbar, O.; Sheehan, J.; Emad, R.; Karim, K.A.; et al. Repeat Stereotactic Radiosurgery for Progressive or Recurrent Vestibular Schwannomas. Neurosurgery 2018, 85, 535-542. [CrossRef] [PubMed]

63. Hong, C.S.; Deng, D.; Vera, A.; Chiang, V.L. Laser-interstitial thermal therapy compared to craniotomy for treatment of radiation necrosis or recurrent tumor in brain metastases failing radiosurgery. J. Neuro-Oncol. 2019, 142, 309-317. [CrossRef]

64. Rae, A.; Gorovets, D.; Rava, P.; Ebner, D.; Cielo, D.; Kinsella, T.J.; DiPetrillo, T.A.; Hepel, J.T. Management approach for recurrent brain metastases following upfront radiosurgery may affect risk of subsequent radiation necrosis. Adv. Radiat. Oncol. 2016, 1, 294-299. [CrossRef]

65. Shehata, W.M.; Hendrickson, F.R.; Hindo, W.A. Rapid fractionation technique and re-treatment of cerebral metastases by irradiation. Cancer 1974, 34, 257-261. [CrossRef]

66. Brown, P.D.; Ballman, K.V.; Cerhan, J.H.; Anderson, S.K.; Carrero, X.W.; Whitton, A.C.; Greenspoon, J.; Parney, I.; Laack, N.N.; Ashman, J.B.; et al. Postoperative stereotactic radiosurgery compared with whole brain radiotherapy for resected metastatic brain disease (NCCTG N107C/CEC·3): A multicentre, randomised, controlled, phase 3 trial. Lancet Oncol. 2017, 18, 1049-1060. [CrossRef]

67. Chang, E.L.; Wefel, J.S.; Hess, K.R.; Allen, P.K.; Lang, F.F.; Kornguth, D.G.; Arbuckle, R.B.; Swint, J.M.; Shiu, A.S.; Maor, M.H.; et al. Neurocognition in patients with brain metastases treated with radiosurgery or radiosurgery plus whole-brain irradiation: A randomised controlled trial. Lancet Oncol. 2009, 10, 1037-1044. [CrossRef]

68. Cohen-Inbar, O.; Tata, A.; Moosa, S.; Lee, C.-C.; Sheehan, J.P. Stereotactic radiosurgery in the treatment of parasellar meningiomas: Long-term volumetric evaluation. J. Neurosurg. 2018, 128, 362-372. [CrossRef]

69. Buchsbaum, J.C.; Suh, J.H.; Lee, S.-Y.; Chidel, M.A.; Greskovich, J.F.; Barnett, G.H. Survival by radiation therapy oncology group recursive partitioning analysis class and treatment modality in patients with brain metastases from malignant melanoma. Cancer 2002, 94, 2265-2272. [CrossRef]

70. Wroński, M.; Maor, M.H.; Davis, B.J.; Sawaya, R.; Levin, V.A. External radiation of brain metastases from renal carcinoma: A retrospective study of 119 patients from the M. D. Anderson Cancer Center. Int. J. Radiat. Oncol. 1997, 37, 753-759. [CrossRef]

71. Sayan, M.; Mustafayev, T.Z.; Sahin, B.; Kefelioglu, E.S.S.; Wang, S.-J.; Kurup, V.; Balmuk, A.; Gungor, G.; Ohri, N.; Weiner, J.; et al. Evaluation of response to stereotactic radiosurgery in patients with radioresistant brain metastases. Radiat. Oncol. J. 2019, 37, 265-270. [CrossRef] [PubMed]

72. Brown, P.D.; Brown, C.A.; Pollock, B.E.; Gorman, D.A.; Foote, R.L. Stereotactic radiosurgery for patients with "radioresistant" brain metastases. Neurosurgery 2008, 62, 656-666. [CrossRef]

73. Selek, U.; Chang, E.L.; Hassenbusch, S.J.; Shiu, A.S.; Lang, F.F.; Allen, P.; Weinberg, J.; Sawaya, R.; Maor, M.H. Stereotactic radiosurgical treatment in 103 patients for 153 cerebral melanoma metastases. Int. J. Radiat. Oncol. 2004, 59, 1097-1106. [CrossRef]

74. Sperduto, P.W.; Kased, N.; Roberge, D.; Xu, Z.; Shanley, R.; Luo, X.; Sneed, P.K.; Chao, S.T.; Weil, R.J.; Suh, J.; et al. Summary Report on the Graded Prognostic Assessment: An Accurate and Facile Diagnosis-Specific Tool to Estimate Survival for Patients With Brain Metastases. J. Clin. Oncol. 2012, 30, 419-425. [CrossRef]

75. Lawrence, Y.R.; Li, X.A.; El Naqa, I.; Hahn, C.A.; Marks, L.B.; Merchant, T.E.; Dicker, A. Radiation Dose-Volume Effects in the Brain. Int. J. Radiat. Oncol. 2010, 76, S20-S27. [CrossRef] 
76. Matsuyama, T.; Kogo, K.; Oya, N. Clinical Outcomes of Biological Effective Dose-Based Fractionated Stereotactic Radiation Therapy for Metastatic Brain Tumors From Non-Small Cell Lung Cancer. Int. J. Radiat. Oncol. 2013, 85, 984-990. [CrossRef] [PubMed]

77. Manning, M.; Cardinale, R.M.; Benedict, S.H.; Kavanagh, B.D.; Zwicker, R.D.; Amir, C.; Broaddus, W.C. Hypofractionated stereotactic radiotherapy as an alternative to radiosurgery for the treatment of patients with brain metastases. Int. J. Radiat. Oncol. 2000, 47, 603-608. [CrossRef]

78. Benedict, S.H.; Yenice, K.M.; Followill, D.; Galvin, J.M.; Hinson, W.; Kavanagh, B.; Keall, P.; Lovelock, M.; Meeks, S.; Papiez, L.; et al. Stereotactic body radiation therapy: The report of AAPM Task Group 101. Med Phys. 2010, 37, 4078-4101. [CrossRef]

79. Timmerman, R.D. An Overview of Hypofractionation and Introduction to This Issue of Seminars in Radiation Oncology. Semin. Radiat. Oncol. 2008, 18, 215-222. [CrossRef]

80. Kim, D.N.; Medin, P.M.; Timmerman, R.D. Emphasis on Repair, Not Just Avoidance of Injury, Facilitates Prudent Stereotactic Ablative Radiotherapy. Semin. Radiat. Oncol. 2017, 27, 378-392. [CrossRef]

81. Hanna, G.; Murray, L.; Patel, R.; Jain, S.; Aitken, K.; Franks, K.; van As, N.; Tree, A.; Hatfield, P.; Harrow, S.; et al. UK Consensus on Normal Tissue Dose Constraints for Stereotactic Radiotherapy. Clin. Oncol. 2018, 30, 5-14. [CrossRef]

82. Grimm, J.; Marks, L.B.; Jackson, A.; Kavanagh, B.D.; Xue, J.; Yorke, E. High Dose per Fraction, Hypofractionated Treatment Effects in the Clinic (HyTEC): An Overview. Int. J. Radiat. Oncol. 2021, 110, 1-10. [CrossRef]

83. Lin, N.U.; Lee, E.Q.; Aoyama, H.; Barani, I.J.; Barboriak, D.P.; Baumert, B.G.; Bendszus, M.; Brown, P.D.; Camidge, D.R.; Chang, S.M.; et al. Response assessment criteria for brain metastases: Proposal from the RANO group. Lancet Oncol. 2015, 16, e270-e278. [CrossRef]

84. Mitsuya, K.; Nakasu, Y.; Horiguchi, S.; Harada, H.; Nishimura, T.; Bando, E.; Okawa, H.; Furukawa, Y.; Hirai, T.; Endo, M. Perfusion weighted magnetic resonance imaging to distinguish the recurrence of metastatic brain tumors from radiation necrosis after stereotactic radiosurgery. J. Neuro-Oncol. 2010, 99, 81-88. [CrossRef]

85. Chernov, M.; Hayashi, M.; Izawa, M.; Ochiai, T.; Usukura, M.; Abe, K.; Ono, Y.; Muragaki, Y.; Kubo, O.; Hori, T.; et al. Differentiation of the Radiation-Induced Necrosis and Tumor Recurrence after Gamma Knife Radiosurgery for Brain Metastases: Importance of Multi-Voxel Proton MRS. min-Minim. Invasive Neurosurg. 2005, 48, 228-234. [CrossRef] [PubMed]

86. Terakawa, Y.; Tsuyuguchi, N.; Iwai, Y.; Yamanaka, K.; Higashiyama, S.; Takami, T.; Ohata, K. Diagnostic Accuracy of 11CMethionine PET for Differentiation of Recurrent Brain Tumors from Radiation Necrosis After Radiotherapy. J. Nucl. Med. 2008, 49, 694-699. [CrossRef] [PubMed]

87. Steindl, A.; Berghoff, A.S. Brain metastases in metastatic cancer: A review of recent advances in systemic therapies. Expert Rev. Anticancer Ther. 2020, 21, 325-339. [CrossRef]

88. Torres-Reveron, J.; Tomasiewicz, H.C.; Shetty, A.; Amankulor, N.M.; Chiang, V.L. Stereotactic laser induced thermotherapy (LITT): A novel treatment for brain lesions regrowing after radiosurgery. J. Neuro-Oncol. 2013, 113, 495-503. [CrossRef] [PubMed] 